\title{
Dispensing with Channel Estimation: Differentially Modulated Cooperative Wireless Communications
}

\author{
Li Wang, Member, IEEE and Lajos Hanzo, Fellow, IEEE
}

\begin{abstract}
As a benefit of bypassing the potentially excessivecomplexity and yet inaccurate channel estimation, differentially encoded modulation in conjunction with low-complexity noncoherent detection constitutes a viable candidate for usercooperative systems, where estimating all the links by the relays is unrealistic. In order to stimulate further research on differentially modulated cooperative systems, a number of fundamental challenges encountered in their practical implementations are addressed, including the time-variant-channel-induced performance erosion, flexible cooperative protocol designs, resource allocation as well as its high-spectral-efficiency transceiver design. Our investigations demonstrate the quantitative benefits of cooperative wireless networks both from a pure capacity perspective as well as from a practical system design perspective.
\end{abstract}

\section{INTRODUCTION}

$\mathbf{T}$ ECHNOLOGICAL advances in integrated circuits and radio-frequency electronics facilitate the employment of ever more sophisticated signal processing and coding algorithms in pursuit of supporting information exchanges between people and/or devices over hostile, band-limited wireless channels, regardless of their geographic positions. Meanwhile, it is increasingly important to find energy- and bandwidth-efficient solutions for next-generation wireless communication, which is limited by classic modulation/transmission schemes. This is because their capacity obeys the Shannon-Hartley law, which only increases the achievable throughput logarithmically with the transmit power. During the late 90's, the emergence of multiple antenna aided multiple-input multipleoutput (MIMO) [1,2] techniques constitutes one of the most significant technical innovations over the decades, rendering the system's capacity linearly dependent on the minimum of the number of the transmit and receive antennas, or equivalently on the transmit power, provided that any extra power is assigned to additional antennas. The research of MIMO systems was inspired by the pioneering work of Foschini [3, 4] and Telatar [5] on the basis of the fundamental philosophy centered around space-time signal processing [6-10], where the natural time-dimension is complemented by the 'spatial' dimension created by the use of multiple spatially distributed

Manuscript received 19 October 2010; revised 19 April 2011 and 18 July 2011. The research leading to these results has received funding from the European Union's Seventh Framework Programme ([FP7/2007-2013]) under grant agreement no [214625]. The finacial support of the RC UK under the auspices of the UK-India Advanced Technology Centre of Wireless Communications and of the China-UK Science Bridge in 4G wireless communications, as well as that of the EU's Concerto project is also gratefully acknowledged.

The authors are with the School of ECS, Universityof Southampton, SO17 1BJ, U.K. (e-mail: 1w5@ecs.soton.ac.uk; 1h@ecs.soton.ac.uk).

Digital Object Identifier 10.1109/SURV.2011.081611.00138 antennas. Specifically, an increased data rate may be attained by multiplexing and simultaneously transmitting different data streams from multiple antennas of a MIMO system. Naturally, the reliability of demultiplexing these streams relies on the separability of the parallel MIMO streams, which may be achieved with the aid of the antenna-specific channel impulse responses (CIRs) in the presence of rich multipath propagation conditions. More explicitly, the CIRs become sufficiently different for distinguishing the MIMO streams for well-separated antennas $[3,11,12]$. On the other hand, the benefits of MIMO systems may also be exploited for mitigating the detrimental effects of multipath propagation with the aid of their transmit/recieve diversity gain, which is an explicit benefit of receiving multiple independently faded transmit signal replicas, in order to improve the attainable robustness $[6,7,10]$. Given a certain target transmission rate, both of the above-mentioned multiplexing and diversity gains may be interpreted as an SNR gain achieved by MIMO systems over their single-input single-output counterparts. The various trade-offs between the multiplexing and diversity have been investigated, for example, in $[13,14]$.

\section{A. Motivations Behind Cooperative Communications}

However, it is often impractical for the pocket-sized mobile device to employ multiple antennas due to its size and cost constraints as well as owing to the associated hardware impairments, such as for example the associated mixed-signal coupling and cross-talk that may become critical in integrated high performance wireless systems, where the digital circuitry is tightly co-located with the analog RF electronics. Furthermore, owing to the limited separation of the antenna elements, the transmitted signal rarely experiences independent fading, in other words the corresponding signal replicas collected at the receiver are more likely to be in a deep fade simultaneously, which in turn erodes the achievable diversity gain. The diversity gain may be further compromised by the deleterious effects of the large-scale shadow fading [15] at high operating frequencies, where all the MIMO channels tend to fade together rather than independently, imposing further signal correlation amongst the antennas in each other's vicinity. Apart from the above obstacles in the way of achieving multipleantenna-aided diversity gains, wireless cellular networks aim for improving the coverage, capacity or the quality of enduser experience (QoE) in inadequately covered areas, such as for example indoor environments and rural areas. The dense deployment of fully-fledged base stations (BSs) constitutes a high-quality solution, albeit this may impose a high infras- 
tructure cost and thus may become economically inviable, especially in low-traffic-density sparsely populated rural areas. Furthermore, in addition to the propagation-loss-induced lowpower reception, the mobile stations (MSs) roaming in the cell edge region may also suffer from severe intercell interference.

Hence, to meet the challenging requirements of nextgeneration wireless networks in terms of coverage, capacity as well as deployment cost, the relay-aided cooperative transmission technique [16-20] appears to be one of the most promising solutions. The idea of cooperative transmission was originally conceived by simply relying on the fundamental broadcast feature of the wireless medium, which is frequently regarded as a drawback. In a nutshell, in multi-user wireless systems, single-antenna-assisted MSs may cooperatively share their antennas in order to achieve the so-called cooperative diversity as well as a path-loss-reduction based power gain by forming a virtual antenna array (VAA) [21,22] in both uplink (UL) and downlink (DL) transmissions. The concept of user cooperation has been first proposed in $[19,20]$ for a twouser cooperative CDMA system, where orthogonal codes are employed by the active users in order to avoid multiple access interference. A user who directly sends his/her own information to the destination is regarded as a source node, while the other users who assist in forwarding the information received from the source node are considered as relay nodes. Naturally, the extra tele-traffic between a source MS and a cooperating MS serving as a relay station (RS) demands additional radio resources to be allocated - any of the well-established multiple access schemes can be employed by the users to guarantee their orthogonal interference-free transmission, such as Time Division Multiple Access (TDMA), Frequency Division Multiple Access (FDMA) or Code Division Multiple Access (CDMA) [17].

\section{B. Cooperative Relaying Protocols and Classification}

The underlying idea behind cooperative transmissions can be traced back to the pioneering work on the information theoretic features of the relay channel [16]. Motivated by this contribution, various cooperation strategies and protocols have been proposed. According to the operations carried out at the RS, the relaying protocols may be classified into three categories, namely amplify-and-forward (AF), decode-andforward (DF) and compress-and-forward (CF) relaying. The former two schemes were devised in [21], which have become the most popular ones because of their simplicity and intuitive designs. In the AF scheme, which is also referred to as the analog-repeater-based arrangement [18], the RS simply amplifies and forwards the source node's 'overheard' signal to the intended destination, thus potentially increasing the system's overall noise level, since the signal and noise are amplified together. As to the DF scheme, the RS fully decodes the signal received from the source and provides the destination with a re-encoded signal. Hence, the problem of error propagation may arise, when the RS forwards the erroneously recovered signal, which may deteriorate the detection at the destination and hence the overall system performance. It was recently demonstrated in $[21,23]$ that the fixed DF system dispensing with any error-aware mechanisms at the RS, such as for

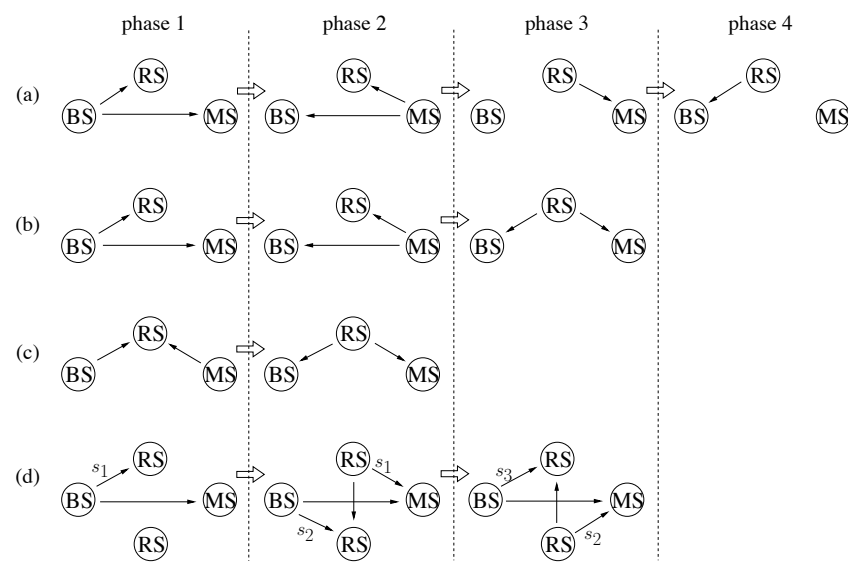

Fig. 1. Relaying Protocols: a) traditional four-phase relaying; b) three-phase relaying; c) two-phase relaying using network coding; d) successive relaying using additional RS.

example, the cyclic redundancy check (CRC) [24], offers no diversity gain over its conventional direct-transmission-based counterpart. Consequently, the selective DF scheme [21,23] was devised with the aid of error detection codes and/or intelligent RS selection schemes, where the RS may forward the signal if and only if it is correctly decoded. Furthermore, when the signal radiated from the RS is encoded to provide extra error protection to the original message, the DF scheme is also known as coded cooperation [25-27]. Recently, the CFbased cooperative scheme also received increasing research attention [28,29], where the RS forwards a quantized or compressed version of the signal received from the source.

On the other hand, based on the time slots required to complete a full cycle of UL and DL transmissions, the family of cooperative relaying systems may be divided into another four subgroups, namely the traditional four-phase mechanisms, the network-coding-aided three-phase and two-phase schemes, as well as the successive relaying strategy, as portrayed in Fig. 1. As demonstrated by Fig. 1(a), the four-phase cooperative scheme, which is also referred to as one-way relaying, may achieve an enhanced transmit diversity gain, beneficial path-loss reductions, as well as the complete orthogonality between the broadcast and relaying phases. As a price to pay for these benefits, the system's effective throughput is halved in comparison to the conventional direct-transmission scheme owing to the half-duplex communications of practical transceivers, which cannot readily transmit and receive simultaneously. Thus, it is hard to formulate an immediate judgement on whether the benefits of user cooperation justify the cost incurred in the interest of increasing the achievable transmission efficiency. For example, recent research in [30] has revealed that the AF-based cooperative system may suffer from a significant capacity loss in comparison to the conventional direct-transmission system. Hence, the three-phase [31, 32] and two-phase [33-35] bidirectional relaying schemes of Figs. 1(b) and 1(c) have been proposed in order to recover the effective throughput reduction, where advanced network coding techniques are employed at the RS to generate and transmit a combined signal stream encapsulating both the DL and UL signals during the relaying phase. As shown by 


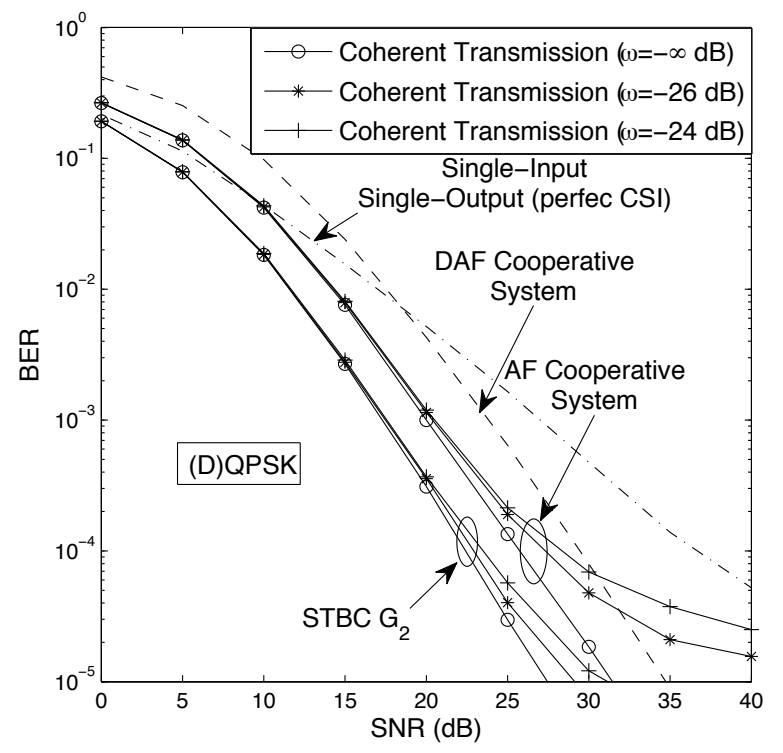

Fig. 2. Performance sensitivity to imperfect channel knowledge of the singlerelay amplify-and-forward cooperative system in conjunction with coherent detection.

Figs 1(b) and 1(c), the two-phase scheme requires less time slots to complete a full cycle of UL and DL transmissions, than its three-phase counterpart at the expense of a typically worse decoding performance at the RS induced by the mutual interference between the UL and DL signals in phase 1 of Fig. 1(c). Recently, the successive relaying technique of Fig. 1(d) has been devised in [36], which needs an additional RS for the sake of recovering the half-duplex-relaying-induced multiplexing loss. The successive relaying carried out by the pair of parallel RSs allows the source to transmit continuously, while still achieving second-order diversity and maintaining almost the same slot efficiency as the direct-transmission system, provided that the number of communications phases is sufficiently high. Furthermore, space-time coding techniques $[6,10,37]$ constitute another spetrally-efficient approach applicable to cooperative systems, leading to the concept of distributed space-time coding schemes [38-41]. For example, each cooperating RS can transmit a column of an orthogonal space-time code matrix during the relaying phase.

\section{Cooperative Systems Using Differential Modulation}

The above distributed VAA formed via user cooperation naturally leads to a number of new challenges, among others synchronization, channel estimation, appropriate cluster formation as well as resource allocation [42-47], some of which will be detailed in this tutorial. In practice, the knowledge of channel state information (CSI) is typically obtained using a channel sounding sequence. Moreover, the estimation of MIMO channels has an exponentially increasing complexity as a function of the number of antennas. Additionally, the relative frequency of estimating the channel has to be increased proportionately to the channel's fluctuation rate characterized by the Doppler frequency. Furthermore, performance degradations may occur when the receiver has imperfect CSI, as illustrated by the BER curve of a $(2 \times 1)$-element $G_{2^{-}}$ aided MIMO system [6] in Fig. 2, where we assume that the channel estimation errors obey the Gaussian distribution and the degree of the CSI estimation errors is governed by the ratio $\omega(\mathrm{dB})$ with respect to the received signal power. Hence, the perfect CSI scenario corresponds to $\omega=-\infty$. To be specific, given a target BER of $10^{-5}$, a performance loss of $5 \mathrm{~dB}$ may be encountered, even when the channel estimation errors are as low as $\omega=-24 \mathrm{~dB}$. What is more, when this second-order transmit diversity achieved by the $G_{2}$ scheme is attained with the aid of a VAA in the context of a single-relay-aided cooperative system, the achievable BER performance may become significantly more sensitive to the imperfect channel knowledge, as also evidenced in Fig. 2. Observe in Fig. 2 that even when the channel estimation errors are as low as $-26 \mathrm{~dB}$, the BER curve of the single-relay-aided AF system tends to level out above $10^{-5}$, thus the secondorder transmit diversity originally achieved in the presence of perfect channel knowledge vanishes. This is because the cooperative system requires the CSI knowledge of both the source-to-relay and relay-to-destination links in comparison to the classic single-phase direct transmission regime of colocated MIMO systems [44,45], whilst it is particularly challenging for the destination to accurately estimate the sourcerelay channel using pilot signal forwarding in the context of AF-based cooperative systems, since the pilots may be further contaminated by relay-induced noise amplification. Based on our above discussion, obtaining sufficiently accurate CSI for cooperative systems may potentially impose both an excessive complexity and a high pilot overhead, especially when the number of cooperating MSs is high and/or when the channel conditions fluctuate relatively rapidly in mobile environments.

Therefore, differentially encoded signaling combined with low-complexity non-coherent detection and thus bypassing the complex yet potentially inaccurate channel estimation process at the receiver becomes an attractive design alternative, leading to differential modulation assisted cooperative communications [48-54]. It is well-recognized that differential modulation, such as the conventional differential phase-shift keying (DPSK) [55], is capable of striking an attractive compromise between the receiver performance attained and the complexity imposed. This is because the corresponding conventional differential detector (CDD) [55] employed at the receiver may extract the data by simply calculating the phase difference between consecutive time samples, provided that the rate of the channel coefficient fluctuation is sufficiently low. Naturally, in the light of the distributed space-time coding principles, the differential space-time coding regime can also be implemented in a distributed manner for user-cooperation aided systems [56-59].

\section{Focus and Outline of the Paper}

In view of the benefits of bypassing the potentially excessive-complexity and yet inaccurate channel estimation, the family of differential modulation schemes combined with non-coherent detection is advocated in this treatise as a viable candidate to be deployed in the context of cooperative systems. Our goal is to stimulate further research on differentially 


\begin{tabular}{c|c|c|c|c|}
\multicolumn{4}{l}{} & \multicolumn{4}{l}{ Frequency } \\
Ch.1 & $T_{1}$ transmits & $T_{2}$ relays $T_{1}$ & $\ldots$ & $T_{M}$ relays $T_{1}$ \\
Ch.2 & $T_{2}$ transmits & $T_{3}$ relays $T_{2}$ & $\ldots$ & $T_{1}$ relays $T_{2}$ \\
$\vdots$ & $\vdots$ & $\vdots$ & $\cdots$ & $\vdots$ \\
Ch.N & $\underbrace{T_{M} \text { transmits }}_{\text {Phase I }}$ & $T_{1}$ relays $T_{M}$ & $\cdots$ & $T_{M-1}$ relays $T_{M}$ \\
\cline { 2 - 4 } & $\underbrace{}_{\text {Phase II }}$ & Time
\end{tabular}

Fig. 3. Channel allocation scheme for the cooperative cluster formed by $M$ MSs in a celluar UL system.

modulated cooperative systems by addressing a number of fundamental challenges encountered in their practical implementations:

- The achievable cooperative diversity gain may significantly deteriorate, when the channel linking the multiple cooperating MSs becomes severely time-selective at high mobile velocities, since the slow-channel-fluctuation prerequisite of facilitating CDD no longer holds.

- The design of flexible cooperative protocols, appropriate cluster formation strategies, as well as a matching cooperative resource allocation procedure is needed in order to further enchance the attainable performance.

- It becomes questionable whether the DF-based relaying system is superior to its direct-transmission counterpart in terms of the maximum achievable spectral efficiency, when the cooperation-induced multiplexing loss is taken into account.

To this end, following a brief overview of the system/channel models employed and of the performance study of Section II, a multiple-channel multiple-symbol (MCMS) joint detection technique is devised for the differentially modulated cooperative system in Section III, in order to mitigate the error floor induced by the time-selective channel. Then, a hybrid cooperative system employing optimized power allocation and relay selection schemes is proposed in Section IV based on a detailed comparative study of differential cooperative systems using the AF and DF protocols. Subsequently, in order to maximize the DDF cooperative system's capacity, the time-resource-allocation (TRA) optimization along with the code-rate-optimization (CRO) schemes are discussed in Section V, followed by the proposal of a practical nearcapacity cooperative system design framework in Section VI. Finally, our concluding remarks are provided in Section VII.

\section{Conventional Differentially Detection Aided COOPERATIVE COMMUNICATIONS}

\section{A. System and Channel Models}

As an example of the channel allocation depicted in Fig. 3 for the cooperative cluster formed by $M$ MSs out of the available cooperating MS candidate pool $\mathcal{P}_{\text {cand }}$ of a cellular UL system, the signal transmission involves two transmission phases, namely, the broadcast phase and the relay phase, which are also referred to as phase I and II. In this treatise, for the sake of simplicity, TDMA as well as FDMA are considered as illustrated in Fig. 3, in order to guarantee orthogonal, i.e. non-interfering transmission amongst cooperating MSs.
Furthermore, since the channel allocation employed for DL and UL transmissions as well as among cooperative users may be deemed to be symmetric, as indicated in Figs. 1(a) and 3, respectively, we focus our attention on the information transmission of a specific MS in the cellular UL scenario of Fig. 4, which may be assisted by $M_{r}=(M-1)$ MSs activated from the set of available cooperating MS candidate pool $\mathcal{P}_{\text {cand }}$ in order to achieve cooperative diversity by forming a VAA. Without loss of generality, we simply assume a single antenna employed by each terminal within the cooperative system, and a total power $P$ shared by the collaborating MSs for transmitting a symbol. Thus, by assuming that $M_{r}$ cooperating MSs are activated out of a total of $\mathcal{P}_{\text {cand }}$, we can express the associated power contraint as: $P=P_{s}+\sum_{m=1}^{M_{r}} P_{r_{m}}$, where $P_{s}$ and $P_{r_{m}}\left(m=1,2, \cdots, M_{r}\right)$ are the transmit power employed by the source MS and the $m$ th RS, respectively. Again, for the sake of simple analytical tractability, we assume that the sum of the distances $D_{s r_{m}}$ between the source MS and the $m$ th RS, as well as that between the $m$ th RS and the BS, which is represented by $D_{r_{m} d}$, is equal to the distance $D_{s d}$ between the source MS and the BS. Additionally, by considering a path-loss exponent of $l$ [15], the average channel power gain $\sigma_{i, j}^{2}$ at the output of the channel can be computed according to the inter-node distance $D_{i, j}$ as $\sigma_{i, j}^{2}=D_{i, j}^{-v}, i, j \in\left\{s, r_{m}, d\right\}$, under the assumption that the channel's power gain at the input of the channel is normalized to unity without loss of generality.

Throughout this treatise we assume that the complexvalued basedband signals undergo Rayleigh fading. In order to provide a good approximation for TDMA-based cooperative systems and to facilitate the study of the non-coherent detection based channel capacity in following sections, we consider a block-fading Rayleigh channel, where the fading coefficients $h_{i, j},\left(i, j \in\left\{s, r_{m}, d\right\}\right)$ are assumed to change in an independent and identically-distributed (i.i.d.) manner from block to block. On the other hand, we consider here a time-selective block-fading model [60], where the channel's envelope exhibits correlation within a transmission block according to the normalized Doppler frequency $f_{d}$ governed by the relative movement of the tranceivers. Unless otherwise specified, the normalized Doppler frequencies exhibited by links of the cooperative system are assumed to be identical to each other for the sake of simplicity.

In order to avoid channel estimation, the source MS differentially encodes its information symbols $v_{s}^{\mathrm{I}}[n] \in \mathcal{M}_{c}=$ $\left\{e^{j 2 \pi m / M_{c}} ; m=0,1, \cdots, M_{c}-1\right\}$, each of which contains $\log _{2} M_{c}$-bit information, as $s_{s}^{\mathrm{I}}[n]=s_{s}^{\mathrm{I}}[n-1] v_{s}^{\mathrm{I}}[n]$. During phase I, the source broadcasts a packet constituted of $L_{p}$ DPSK symbols $s_{s}^{\mathrm{I}}[n], \quad\left(n=0,1, \cdots, L_{p}-1\right)$, while the BS as well as the RSs receive and store it. In the ensuing phase II, the differential AF (DAF) or the differential DF (DDF) scheme is employed by the RSs, which is the differentially modulated version of the protocols proposed in [21].

Specifically, the signal received at the BS during phase I may be expressed for both the DAF- and DDF-aided systems as:

$$
y_{d}^{\mathrm{I}}[n]=\sqrt{P_{s}} s_{s}^{\mathrm{I}}[n] h_{s d}[n]+w_{d}^{\mathrm{I}}[n] .
$$

Moreover, for the DAF-aided system, the signal received at 


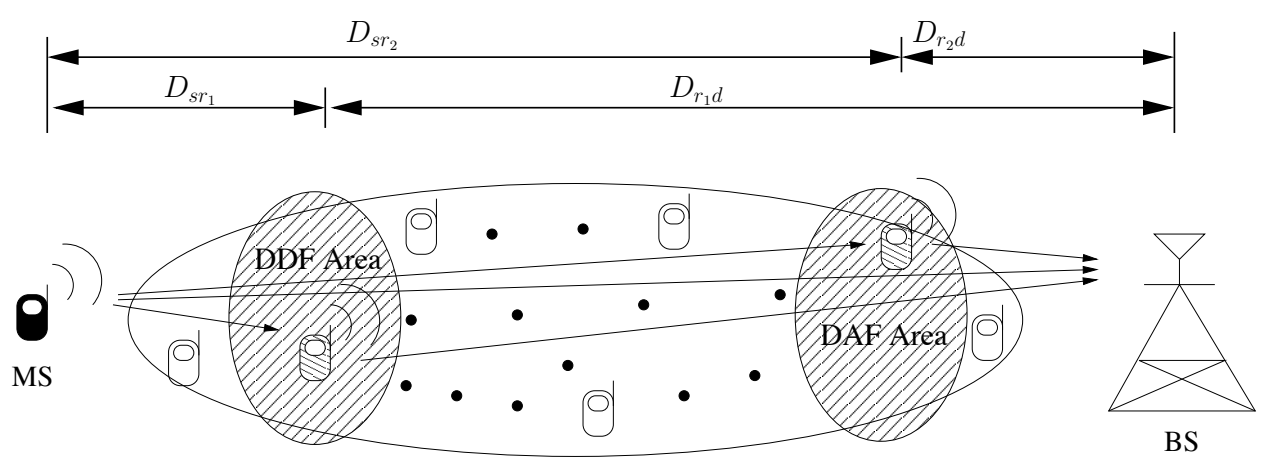

Available Cooperative MS Pool

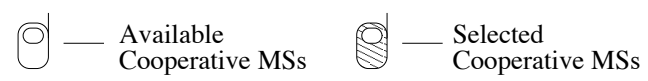

Fig. 4. Cooperation-aided cellular uplink using cooperating-user-selection.

the $m$ th RS during phase I, and that arriving from the $m$ th $\mathrm{RS}$ at the BS during phase II, can be represented as:

$$
\begin{aligned}
y_{r_{m}}^{\mathrm{I}}[n] & =\sqrt{P_{s}} s_{s}^{\mathrm{I}}[n] h_{s r_{m}}[n]+w_{r_{m}}^{\mathrm{I}}[n], \\
y_{d}^{\mathrm{II}}\left[n+m L_{p}\right] & =f_{A M_{r_{m}}} y_{r_{m}}^{\mathrm{I}}[n] h_{r_{m} d}\left[n+m L_{p}\right] \\
& +w_{d}^{\mathrm{II}}\left[n+m L_{p}\right],
\end{aligned}
$$

respectively, where the amplification factor [61] $f_{A M_{r_{m}}}$ used by the $m$ th RS can be computed as $f_{A M_{r_{m}}}=\sqrt{\frac{P_{r_{m}}}{P_{s} \sigma_{s r_{m}}+N_{0}}}$, where $N_{0}=2 \sigma_{w}^{2}$ is the variance of the AWGN $w_{i},(i \in s, r, d)$ imposed at all cooperating MSs as well as at the BS.

As to the DDF-aided system, the RS checks with the aid of CRC codec [24] whether the current packet was error-free and if so, the activated RS forwards the packet received. This allows us to avoid the potential error propagation. Thus, in case of perfect signal recovery, the signal received from the $m$ th RS at the BS during phase II can be expressed as:

$$
\begin{gathered}
y_{d}^{\mathrm{II}}\left[n+m L_{p}\right]=\sqrt{P_{r_{m}}} s_{r_{m}}^{\mathrm{II}}[n] h_{r_{m} d}\left[n+m L_{p}\right] \\
+w_{d}^{\mathrm{II}}\left[n+m L_{p}\right] .
\end{gathered}
$$

Finally, the BS carries out the maximum ratio combining (MRC) $[48,55,62]$ of the signal received directly from the source and those gleaned from all the activated RSs, followed by the CDD process operating without acquiring any CSI. To be specific, based on the multichannel differential detection principle of [55], the BS combines all the signals arriving from the source MS and multiple activated RSs as:

$$
\begin{aligned}
y & =a_{0}\left(y_{d}^{\mathrm{I}}[n-1]\right)^{*} y_{d}^{\mathrm{I}}[n] \\
& +\sum_{m=1}^{M_{r}} a_{m}\left(y_{d}^{\mathrm{II}}\left[n+m L_{p}-1\right]\right)^{*} y_{d}^{\mathrm{II}}\left[n+m L_{p}\right],
\end{aligned}
$$

where $a_{0}$ and $a_{m}\left(m=1,2, \cdots M_{r}\right)$ are the corresponding weighting coefficients, the MRC-based DAF- and DDFaided systems are documented in [48]. By assuming that the channel coefficients $h_{s r_{m}}$ as well as $h_{r_{m} d}$ are near-constant for two successive symbol periods, the BS carries out CDD based on the combined signal $y$ of (5) as $e^{j 2 \pi \hat{m} / M_{c}}=$ $\arg \max _{\check{m}=0,1, \cdots, M_{c}-1} \Re\left\{e^{-j 2 \pi \check{m} / M_{c}} y\right\}$, where $\Re\{\cdot\}$ denotes the real component of a complex number.

\section{B. Classic Unified Approach to Performance Analysis}

A unified approach to the evaluation of the probability of error for differentially modulated direct-transmission based systems discussed in [63] is also applicable to the theoretical bit-error-rate (BER) performance analysis of both the DAFand DDF-aided cooperative systems, which is summarized as follows:

Step 1: Determine the error probability expression conditioned on the received SNR per bit. According to [63], under the assumption that $M_{r}$ RSs are activated in order to forward the source MS's signal to the BS, the DPSK modulated cooperative system's conditional BER may be expressed as:

$$
P_{B E R \mid \gamma^{b}}=\frac{1}{4^{\left(M_{r}+1\right)} \pi} \int_{-\pi}^{\pi} f\left(\beta, M_{r}+1, \theta\right) e^{-\alpha(\theta) \gamma^{b}} d \theta
$$

where $\alpha(\theta)$ and $f(\cdot, \cdot, \cdot)$ are given by (11) and (12) of [48], respectively. Furthermore, $\beta$ is the modulation-dependent factor defined in [63].

Step 2: Formulate the received SNR per bit. Since the MRC scheme is employed at the BS, the SNR per bit at the output of the MRC is given by the sum of the received SNR per bit of each link, where we have $\gamma^{b}=$ $\gamma_{s d}^{b}+\sum_{m=1}^{M_{r}} \gamma_{r_{m} d}^{b}$ for a specific instant when $M_{r}$ RSs are participating in the signal's relaying. Both $\gamma_{s d}^{b}$ and $\gamma_{r_{m} d}^{b}$ may be readily obtained based on (1)-(4), which are both functions of the corresponding channel gains [64]. Specifically, in the light of (1)-(5) and using the MRC-based combining coefficients $a_{i}$ given in [48], we may arrive at $\gamma^{b}=$ $\frac{1}{\log _{2} M c}\left(\frac{P_{s}\left|h_{s d}\right|^{2}}{N_{0}}+\sum_{m=1}^{M_{r}} \frac{P_{s} P_{r_{m}}\left|h_{s r_{m}}\right|^{2}\left|h_{r_{m} d}\right|^{2}}{N_{0}\left(P_{s} \sigma_{s r_{m}}^{2}+P_{r_{m}}\left|h_{r_{m}}\right|^{2}+N_{0}\right)}\right)$ and $\gamma^{b}=\frac{1}{\log _{2} M c}\left(\frac{P_{s}\left|h_{s d}\right|^{2}}{N_{0}}+\sum_{m=1}^{M_{r}} \frac{P_{r}\left|h_{r d}\right|^{2}}{N_{0}}\right)$ for the DAF- and the DDF-aided systems, respectively, under the assumption that $M_{r}$ RSs are participating in relaying the source MS's signal.

Step 3: Average the above-mentioned conditional error probability $P_{B E R \mid \gamma^{b}}$ over the entire range of $\gamma^{b}$ values by weighting it according to its probability of occurrence with the aid of its probability density function (PDF). Note that under the assumption of Rayleigh fading channels, the PDF of the instantaneous received SNR per bit of each link is subject to the so-called $\Gamma$ distribution [15].

Following the method outlined above, a high-SNR-based 


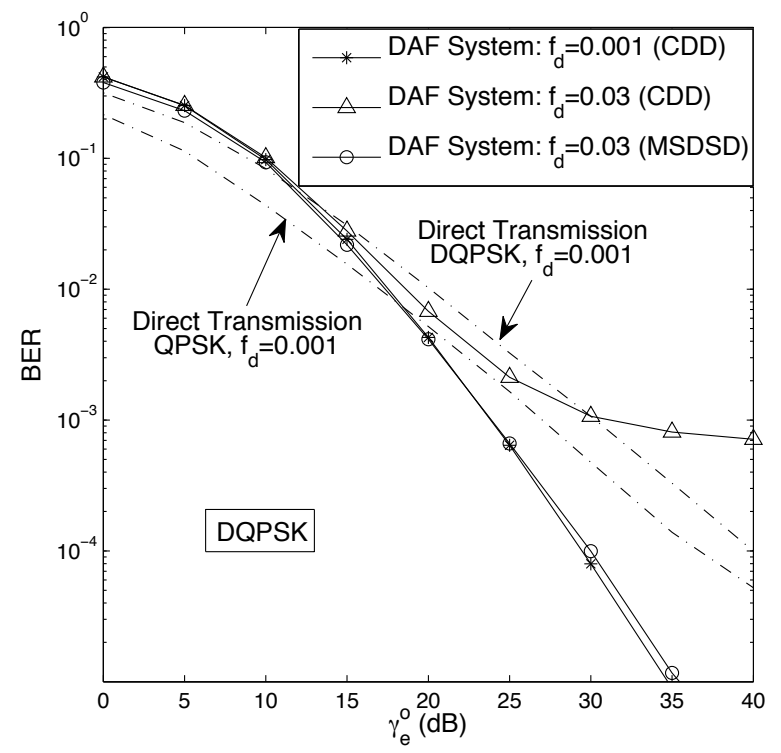

Fig. 5. Impact of user mobility on the performance of the DAF-aided cooperative system using the CDD. $\left(v=3, M_{r}=2, D_{s r_{m}}=\frac{1}{2} D_{s d}\right.$, $P_{s}=P_{r m}=P /\left(M_{r}+1\right), m=1,2, \cdots, M_{r}, N_{\text {wind }}=11$ is employed by the MSDSD)

asymptotic BER curve can be obtained for both the DAF- and DDF-aided cooperative systems, for example, as derived in $[48,49,64]$. As will be seen in Section IV, the approximate BER expression derived may be rather useful in analyzing the resource allocation of cooperative systems, since it allows us to numerically calculate the achievable BER corresponding to various resource allocation arrangements.

\section{Drawbacks of CDD-Aided Differential Transmission}

1) Inherent $3 d B$ Performance Loss: As discussed in Section II-A, since the data is encoded as the phase difference between the two successively transmitted symbols by the conventional DPSK signalling process, the CDD employed at the receiver is capable of recovering the information by directly calculating the phase difference of the two consecutively received symbols, under the assumption that the fading coefficients remain almost unchanged over the corresponding two adjacent symbol durations. Based on this differential encoding and decoding mechanism, it is intuitive that in the CDD-aided direct-transmission system, any received symbol that has been heavily noise-contaminated is likely to cause errors in recovering its related two consecutively differentially encoded symbols. In other words, the differentially modulated direct transmission detected by the CDD scheme may circumvent the channel estimation at the expense of doubling the equivalent noise power, which in turn leads to the well-known $3 \mathrm{~dB}$ performance loss in comparison to its coherent-detectionaided counterpart in the context of slow fading channels, for example, when experiencing a normalized Doppler frequency of $f_{d}=0.001$, as indicated by the gap between the dash-dotted lines in Fig. 5, where BER performance is plotted against the system's overall equivalent $\mathrm{SNR}^{1}$.

2) Detrimental Effects of High Mobile Velocity: When the channel linking the multiple terminals becomes more time-selective at high mobile velocities, the slow-channelfluctuation prerequisite to carry out the CDD no longer holds. Hence, a potentially significant performance degradation is expected for the CDD-aided differential direct transmissions, which implies that the cooperative diversity gains achieved by the CDD-aided cooperative system may also decrease, as the relative mobile velocities of the cooperating users with respect to both each other and to the BS increase. For example, observe in Fig. 5 that the transmit diversity gain achieved by the single-relay-aided DAF system is significantly reduced as the normalized Doppler frequency changes from $f_{d}=0.001$ to $f_{d}=0.03$. As a result, the BER curve levels out just below $10^{-3}$, as the SNR increases.

\section{Multiple-Channel Multiple-Symbol Joint DETECTION FOR DIFFERENTIAL MODULATED COOPERATIVE SYSTEM}

In order to eliminate the above performance degradation in highly mobile environments and still achieve full cooperative diversity without the channel estimation for differentially modulated cooperative systems, joint detection of the signals arriving from the source MS and the cooperating RSs within an observation window spanning $N_{\text {wind }}$ symbol durations is proposed at the BS. Hence, the receiver becomes capable of exploiting the correlation between the phase distortions experienced by the consecutive transmitted user-cooperation based space-time symbols, which is defined as a $\left(M_{r}+1\right) \times\left(M_{r}+1\right)$ dimensional diagonal matrix $\mathbf{S}_{n}=\operatorname{diag}\left\{\left[s_{s}^{\mathrm{I}}[n], \cdots, s_{s}^{\mathrm{I}}[n]\right]\right\}$. In other words, the BS makes a decision about a block of $\left(N_{\text {wind }}-1\right)$ consecutive user-cooperation based space-time symbols relying on collecting $N_{\text {wind }}$ user-cooperation-based received signal vectors $\mathbf{y}_{n}=\left[y_{d}^{\mathrm{I}}[n] y_{d}^{\mathrm{II}}\left[n+L_{p}\right] \cdots y_{d}^{\mathrm{II}}[n+\right.$ $\left.\left.\left(M_{r}-1\right) L_{p}\right]\right]^{T}$, thus enabling the detector to exploit the second-order statistics of the fading channels, which is assumed to be known. Ideally, the error floor encountered by the differentially modulated cooperative system when performing CDD as observed in Fig. 5 can be essentially eliminated, provided that the value of $N_{\text {wind }}$ is sufficiently high. In our ensuing discourse, a generalized equivalent multiple-channel multiple-symbol (MCMS) based transmission model will be constructed in Section III-A. Then, based on this MCMS scheme, the design of multiple-symbol-based non-coherent detection conceived for the differential cooperative system will be detailed in Section III-B.

\section{A. Generalized Equivalent Multiple-Channel Multiple-Symbol Based Transmission Model}

A generalized equivalent MCMS based transmission model for both the DAF- and DDF-aided cooperative systems may be formulated as follows:

$$
\underline{\mathbf{y}}=\underline{\mathbf{S h}}+\underline{\mathbf{w}},
$$

\footnotetext{
${ }^{1}$ The terminology of 'equivalent SNR' is used here to indicate the fact that it quantifies the ratio of the transmit power and the receiver's noise, which are measured at physically different points. This is in line with [65] for example $\gamma_{e}^{o}=P / 2 \sigma_{w}^{2}$ represents the network's overall equivalent SNR.
} 
where the vector of received signals $\mathbf{y}$ is constructed by vertically concatenating $N_{\text {wind }}$ consecutively received usercooperation based symbol vectors $\mathbf{y}_{n}$. Accordingly, the channel's corresponding block vector $\underline{\mathbf{h}}$ as well as the AWGN block vector $\underline{\mathbf{w}}$ are also defined by vertically concatenating the $N_{\text {wind }}$ corresponding user-cooperation based component vectors $\mathbf{h}_{n}$ and $\mathbf{w}_{n}$, respectively. In the light of (1)-(4), the expressions of $\mathbf{h}_{n}$ and $\mathbf{w}_{n}$ may be readily obtained for both the DAFand DDF-aided systems. Note that for the DAF-aided system, the relay-link-related components in $\mathbf{h}_{n}$ and $\mathbf{w}_{n}$ are products of two complex Gaussian variables, as derived in [66]. Moreover, the diagonal block matrix of the transmitted signal is constructed as $\underline{\mathbf{S}}=\operatorname{diag}\left\{\mathbf{S}_{n}, \mathbf{S}_{n+1}, \cdots, \mathbf{S}_{n+N_{\text {wind }}-1}\right\}$.

\section{B. Multiple-Symbol Differential Sphere Detection Design for Differentially Modulated Cooperative Systems}

It is worth emphasizing that all the elements in $\underline{\mathbf{h}}$ and $\underline{\mathbf{w}}$ exhibit a standard Gaussian distribution for the DDF-aided cooperative system, but not for its DAF-aided counterpart. However, intensive simulation-based investigations of [66] suggest that the resultant noise processes are near-Gaussian distributed. As a result, the PDF of the received signal vector $\mathbf{y}$ in (7) is also near-Gaussian, especially for relatively low SNRs, where the effects of the AWGN become more dominant. Hence, under the simplifying assumption that the equivalent fading and noise are zero-mean complex Gaussian processes, the PDF of the non-coherent receiver's output $\underline{\mathbf{y}}$ in (7) conditioned on the transmitted signal vector $\mathbf{s}_{s}^{\mathrm{I}}=$ $\left[s_{s}^{\mathrm{I}}[n], s_{s}^{\mathrm{I}}[n+1], \cdots, s_{s}^{\mathrm{I}}\left[n+N_{\text {wind }}-1\right]\right]$ may be approximately expressed as follows for both the DAF- and DDF-aided scenarios:

$$
p\left(\underline{\mathbf{y}} \mid \mathbf{s}_{s}^{\mathrm{I}}\right) \approx \frac{\exp \left(-\underline{\mathbf{y}}^{H}\left(\Psi\left(\mathbf{s}_{s}^{\mathrm{I}}\right)\right)^{-1} \underline{\mathbf{y}}\right)}{\operatorname{det}(\pi \Psi)},
$$

where we have $\Psi\left(\mathbf{s}_{s}^{\mathrm{I}}\right)=\mathcal{E}\left\{\mathbf{y} \mathbf{y}^{H} \mid \mathbf{s}_{s}^{\mathrm{I}}\right\}=\underline{\mathbf{S}} P \Sigma_{h} \underline{\mathbf{S}}^{H}+$ $2 \sigma_{w}^{2} \mathbf{I}_{N_{\text {wind }}}$ with $\Sigma_{h}=\mathcal{E}\left\{\underline{\mathbf{h}}^{H}\right\}$ representing the channel covariance matrix and $\mathbf{I}_{N_{\text {wind }}}$ denoting the $\left(N_{\text {wind }} \times N_{\text {wind }}\right)$ element identity matrix. With the aid of Bayes' theorem, the decision metric of the maximum-likelihood multiplesymbol differential detection (ML-MSDD) designed for the differential cooperative system may be expressed as $\hat{\mathbf{s}}_{s \mathrm{IL}}^{\mathrm{I}}=$

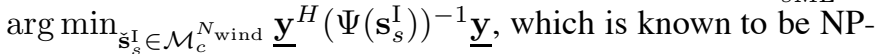
hard. Hence, a pontentially excessive computational complexity may be imposed. For example, under the assumption of an observation window size of $N_{\text {wind }}=10$ and that of DQPSK $\left(M_{c}=4\right), 2^{20}=1.048576 \times 10^{6}$ legitimate user-cooperation based space-time constellation points have to be checked, thus precluding the practical implementation of the ML-MSDD at the BS of our differentially encoded non-coherent cooperative system. As a remedy, the classic sphere detection (SD) algorithm may be invoked, which was originally derived by Pohst and Finke [67] for efficiently calculating a vector of short length in a lattice. The SD was then further developed for coherent-detection-based communication systems [68] by Viterbo and Boutros. As a result, the coherent ML performance is approached at a moderate complexity, which is polynomially, rather than exponentially dependent on the number of unknowns. Inspired by above
TABLE I

MAJOR CONTRIBUTIONS ON DIFFERENTIAL SPHERE DETECTION.

\begin{tabular}{|c|c|}
\hline $\begin{array}{l}\text { [69] Lampe et al. } \\
\text { (2005) }\end{array}$ & $\begin{array}{l}\text { The first paper to introduce the SD algo- } \\
\text { rithm to mitigate the complexity of ML- } \\
\text { MSDD of }[70,71] .\end{array}$ \\
\hline $\begin{array}{l}\text { [79] Pauli and Lampe } \\
\text { (2005) }\end{array}$ & $\begin{array}{l}\text { The first contribution to extend the } \\
\text { MSDSD to detect the differential space- } \\
\text { time modulation. }\end{array}$ \\
\hline $\begin{array}{l}\text { [80] Pauli et al. } \\
\text { (2006) }\end{array}$ & $\begin{array}{l}\text { A soft-decision-aided MSDSD is devised, } \\
\text { which can be employed in iterative detec- } \\
\text { tion assisted receivers. }\end{array}$ \\
\hline $\begin{array}{l}\text { [81] Pauli and Lampe } \\
\text { (2007) }\end{array}$ & $\begin{array}{l}\text { Complexity of the MSDSD is intensively } \\
\text { investigated in the paper. }\end{array}$ \\
\hline $\begin{array}{l}\text { [82] Pauli et al. } \\
\text { (2008) }\end{array}$ & $\begin{array}{l}\text { 2-D observation window technique is con- } \\
\text { trived for the MSDSD employed in the } \\
\text { MIMO-OFDM system using differential } \\
\text { space-frequency modulation. }\end{array}$ \\
\hline $\begin{array}{c}\text { [66] Wang and Hanzo } \\
\text { (2009) }\end{array}$ & $\begin{array}{l}\text { The first paper to specifically design the } \\
\text { MSDSD for the differential cooperative } \\
\text { system. }\end{array}$ \\
\hline
\end{tabular}

contributions, the SD algorithm was first introduced by Lampe et al. in [69] for mitigating the complexity of the ML-MSDD $[70,71]$ in the context of a differentially modulated directtransmission based system, leading to the multiple-symbol differential sphere detection (MSDSD) concept. In the light of this, the SD algorithm may also be introduced to tackle the above-mentioned complexity problem of the ML-MSDD scheme proposed for our differential cooperative system. In the interest of ease of presentation, the mathematical details are omitted here, but the resultant ML-MSDSD based decision metric may be formulated as [66]:

$$
\begin{aligned}
\hat{\mathbf{s}}_{s \mathrm{ML}}^{\mathrm{I}} & =\arg \min _{\check{\mathbf{s}}_{s}^{\mathrm{I}} \in \mathcal{M}_{c}^{N_{\text {wind }}}}\left\|\mathbf{U} \check{\mathbf{s}}_{s}^{\mathrm{I}}\right\|^{2} \\
& =\arg \min _{\check{\mathbf{s}}_{\mathbf{s}}^{\mathrm{I}} \in \mathcal{M}_{c}^{U}} \sum_{i=1}^{U} \underbrace{u_{i i}^{2}\left[\check{s}_{s i}^{\mathrm{I}}+\sum_{j=i+1}^{U} \frac{u_{i j}}{u_{i i}} \check{s}_{s j}^{\mathrm{I}}\right]^{2}}_{\phi_{i}},
\end{aligned}
$$

where $\mathbf{U}$ is an upper-triangular matrix, which can be obtained as $\mathbf{U} \triangleq(\mathbf{F} \operatorname{diag}\{\mathbf{y}\})^{*}$, with $\mathbf{F}$ also being an upper-triangular matrix generated using the well-known Cholesky factorization [72] of the matrix $\left(\Sigma_{h}+2 \sigma_{w}^{2} \mathbf{I}_{N_{w i n d}}\right)^{-1}$. Consequently, thanks to the upper-triangular structure of the matrix $\mathbf{U}$, a layered tree search may be carried out within an increasingly smaller hyper-spheric search space. Table I summarizes the major contributions addressing the design of the MSDSD scheme for non-coherent transmissions. The interested reader is referred to [73-76] and the references therein for a more comprehensive treatment of the SD algorithms. Although our attention is focused on the MSDSD in this treatise, it is worth noting that mechanisms other than that of the SD scheme can be also employed to achieve a beneficial complexity reduction for the MSDD, such as for example discussed in [77,78].

\section{An Application Example}

As an application example, the MSDSD designed in conjunction with $N_{\text {wind }}=11$ is employed at the BS to mitigate the detrimental effects imposed by the highly mobile environment for the single-relay-aided DAF system. As observed in 


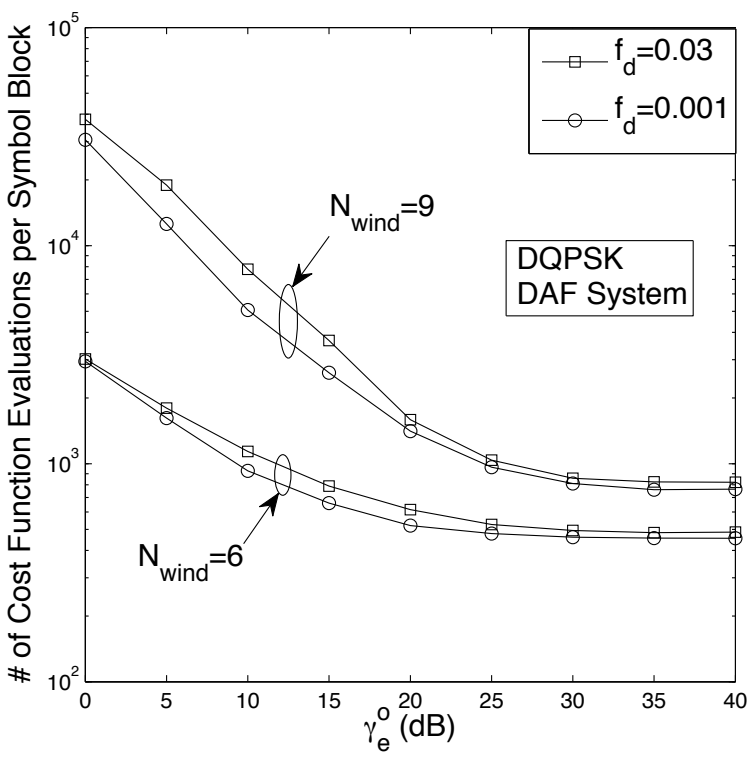

Fig. 6. Complexity imposed by the MSDSD versus the SNR in the singlerelay-aided DAF cooperative system.

Fig. 5, the error floor encountered by the system employing the CDD in time-selective fading channels was essentially eliminated with the aid of the MSDSD. This was achieved by circumventing the ML-search of $2^{\left(M_{c} \times N_{\text {wind }}\right)}$ decision candidates with the aid of the MSDSD at a substantially reduced computational complexity. More specifically, the BER curve corresponding to the MSDSD-aided cooperative system generated for $f_{d}=0.03$ coincides with that of its CDD-aided counterpart, which was recorded for a slow-fading channel associated with $f_{d}=0.001$. Hence our solution resulted in a performance gain of more than $10 \mathrm{~dB}$ at a target BER of $10^{-3}$.

The complexity quantified by the number of cost function evaluations, namely $\phi_{i}$ of (9), imposed by the MSDSD versus the SNR is plotted in Fig. 6, where the complexity curves corresponding to $N_{\text {wind }}=9$ are evidently above those corresponding to $N_{\text {wind }}=6$. Moreover, the complexity imposed by the MSDSD decreases steadily, as the SNR increases and finally levels out in the high-SNR range. This is not unexpected, since under the assumption of having a reduced noise contamination, it is more likely that the ML solution point $\hat{\mathbf{s}}_{s \mathrm{ML}}^{\mathrm{I}}$ is located near the search center of the SD used for finding the ML-MSDD solution. As a result, the SD's search process may converge much more rapidly, imposing a reduced complexity. Furthermore, we can also observe from Fig. 6 that the Doppler frequency has a non-negligible effect on the complexity imposed by the MSDSD. Basically, for a constant value of $N_{\text {wind }}$, a reduced grade of channel predictability associated with an increased Doppler frequency may lead to an increased complexity imposed by the MSDSD scheme.

\section{The Resource-Optimized DifFERENTIALLY Modulated CoOperative Cellular Uplink}

Although a full spatial diversity may now be maintained by employing the MSDSD for both the DAF- and DDF- aided cooperative systems, the achievable end-to-end BER performance may significantly depend both on the specific choice of the cooperative protocol employed and/or on the quality of the relay channel. Therefore, in the scenario of the differentially modulated cooperative uplink, where multiple cooperating MSs are roaming in the area between a specific MS and the BS, an appropriate and flexible cooperative protocol as well as a matching cooperative resource allocation procedure becomes necessary in order to maintain a desirable end-to-end performance.

\section{A. Resource Optimization for the Cooperative Uplink}

As mentioned in Section II, with the aid of the theoretical BER bound expressions derived for the DAF- and DDFassisted cooperative uplinks, both the transmit power and the RS locations can be optimized by using the minimum BER criterion. Specifically, the optimized location of the cooperating RSs, expressed in terms of the normalized distance $d_{m}=D_{s r_{m}} / D_{s d},\left(m=1,2, \cdots, M_{r}\right)$ between the source MS and the RS, can be found numerically based on the theoretical BER bound expression for a given power allocation $c_{m}=P_{r_{m}} / P_{s}$, and vice versa. However, for the sake of attaining the globally optimum resource allocation solution, which corresponds to the lowest point of the BER surface of Fig. 7(a), an iterative power-versus-RS-location optimization process has to be performed, which is characterized by the step-by-step optimization trajectory seen in Fig. 7(b). This procedure is summarized as follows:

Step 1: Initialize the starting point $\left(\left\{c_{m}\right\}_{m=1}^{M_{r}},\left\{d_{m}\right\}_{m=1}^{M_{r}}\right)$ for the search in the $2 M_{r}$-dimensional space, hosting the $M_{r}$ powers and RS locations.

Step 2: Calculate the locally optimum location $\left\{d_{m, l o c a l}\right\}_{m=1}^{M_{r}}$ of the cooperating users for the current power allocation, $\left\{c_{m}\right\}_{m=1}^{M_{r}}$.

Step 3: If we have $\left\{d_{m, \text { local }}\right\}_{m=1}^{M_{r}} \neq\left\{d_{m}\right\}_{m=1}^{M_{r}}$, then let $\left\{d_{m}\right\}_{m=1}^{M_{r}}=\left\{d_{m, \text { local }}\right\}_{m=1}^{M_{r}}$. Otherwise, stop the search, since the globally optimum solution has been found: $\left\{d_{m, \text { globle }}\right\}_{m=1}^{M_{r}}=\left\{d_{m, \text { local }}\right\}_{m=1}^{M_{r}}$ and $\left\{c_{m, \text { globle }}\right\}_{m=1}^{M_{r}}=$ $\left\{c_{m}\right\}_{m=1}^{M_{r}}$.

Step 4: Calculate the locally optimum power allocation $\left\{c_{m, \text { local }}\right\}_{m=1}^{M_{r}}$ of the cooperating RSs for the current location, $\left\{d_{m}\right\}_{m=1}^{M_{r}}$.

Step 5: If we have $\left\{c_{m, l o c a l}\right\}_{m=1}^{M_{r}} \neq\left\{c_{m}\right\}_{m=1}^{M_{r}}$, then let $\left\{c_{m}\right\}_{m=1}^{M_{r}}=\left\{c_{m, l o c a l}\right\}_{m=1}^{M_{r}}$ and return to Step1. Otherwise, stop the search, since the globally optimum solution has been found: $\left\{d_{m, \text { globle }}\right\}_{m=1}^{M_{r}}=\left\{d_{m, \text { local }}\right\}_{m=1}^{M_{r}}$ and $\left\{c_{m, \text { globle }}\right\}_{m=1}^{M_{r}}=\left\{c_{m}\right\}_{m=1}^{M_{r}}$.

Without loss of generality, we simply assume that the locations of all the cooperating candidates are independent and uniformly distributed along the direct line-of-sight (LOS) link connecting the source MS and the BS, which are expected to change from time to time. Since it is likely that no available cooperating MS candidate is situated in the exact optimum location found by the above-mentioned optimization procedure, our proposed cooperating-user-selection (CUS) scheme simply activates (under the assumption that the BS has the knowledge of the user's location) the specific MS roaming closest to the optimum location, $\left\{d_{m, \text { globle }}\right\}_{m=1}^{M_{r}}$, which is calculated 


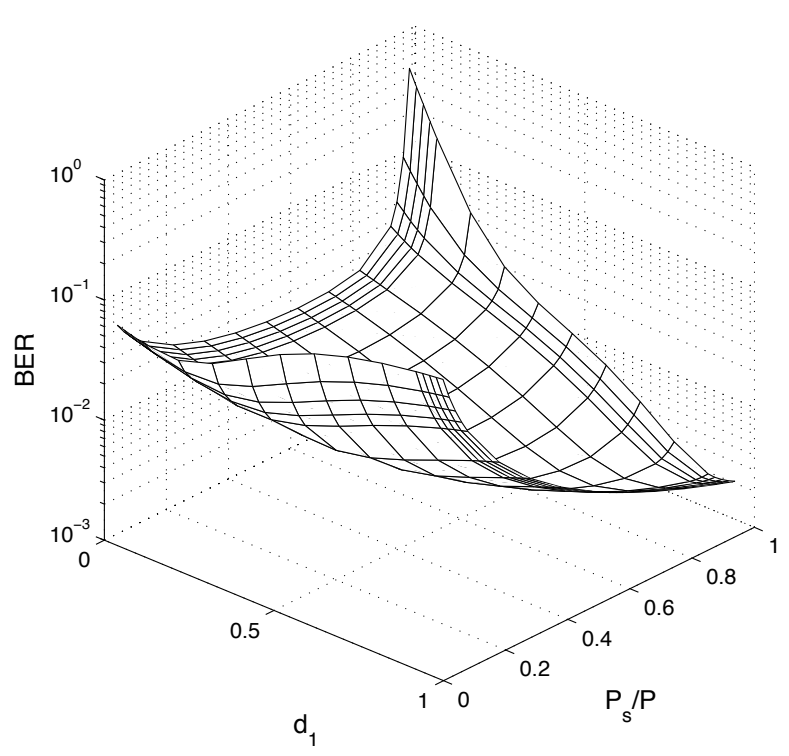

(a) BER surface

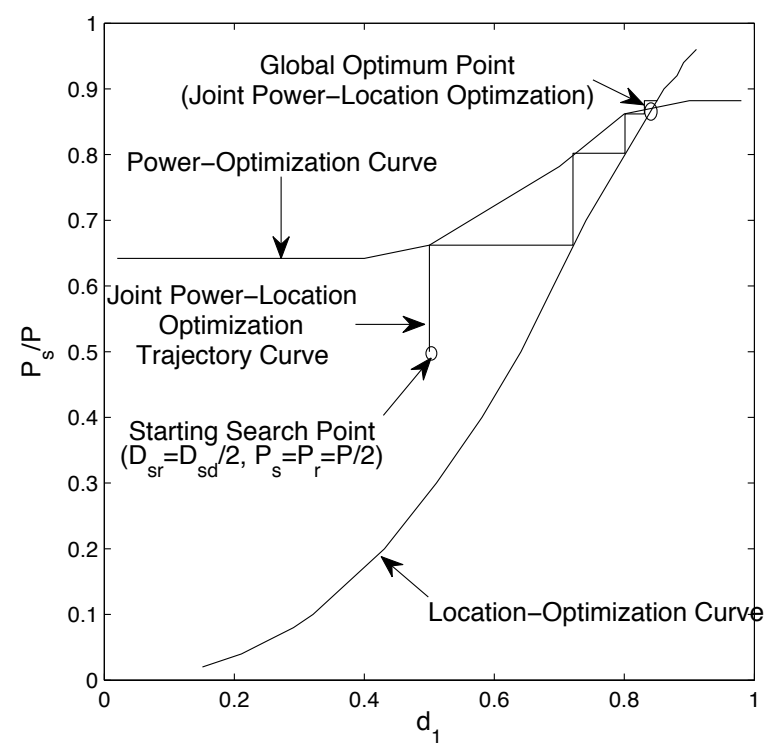

(b) Search Trajectory

Fig. 7. Optimum cooperative resource allocation for DQPSK modulated DAF-aided cooperative cellular systems having a single activated cooperating $\mathrm{MS}$ at $\mathrm{SNR}=15 \mathrm{~dB}$.

off-line with the aid of the above-mentioned iterative powerversus-RS-location optimization process. The rationale of the CUS scheme is based on the observation that the achievable BER is proportional to the distance between the cooperating MS and the optimum location, as indicated in Figs. 9(a) and 10(a), where the BER is portrayed versus $d_{m}$ for both the DAF- and DDF-aided cooperative uplinks activating $M_{r}$ cooperating RSs. Then, the cooperating cluster may adaptively adjust its power-sharing arrangement according to the activated RS's actual location. In our following discourse, we will detail the benefits of the minimum-BER-based resource allocation using the simple application example of a DAF cooperative system in conjunction with the adaptive power allocation (APA) and RS selection. Fig. 8(a) plots the BER curves corresponding to different cooperating MS candidate pool sizes $\mathcal{P}_{\text {cand }}$ versus the overall equivalent SNR $\gamma_{e}^{o}$ for the DAF-aided uplink, where $M_{r}=3$ out of $\mathcal{P}_{\text {cand }}$ cooperating MSs are activated. Interestingly, despite having a fixed number of activated cooperating MSs, upon increasing the value of $\mathcal{P}_{\text {cand }}$, the end-to-end BER performance of the DAF-aided system steadily improves and approaches that of the idealized benchmark system, where an infinite number of cooperating candidates are assumed to be independently and uniformly distributed between the source MS and the BS, as observed in Fig. 8(a). On the other hand, it can be seen in Fig. 8(b) that the higher the number of cooperating candidates, the lower the computational complexity imposed by the MSDSD at the BS. Specifically, observe in Fig. 8 that by increasing the size of the candidate pool from $\mathcal{P}_{\text {cand }}=3$ to 9 , a performance gain of about $7 \mathrm{~dB}$ can be attained, while simultaneously achieving a detection complexity reduction factor of 6.5 at the target BER of $10^{-5}$. In comparison to the idealized scenario, the DAFaided cooperative system using the proposed low-complexity resource allocation scheme only suffers a negligible performance loss, when having $\mathcal{P}_{\text {cand }}=9$ cooperating candidates. Therefore, the benefits brought about by the employment of the power allocation and RS selection may be deemed substantial in a typical cellular uplink, as manifested by our simple application example.

\section{B. Comparison of the DAF- and DDF-Aided Systems}

The BER performance of both the DAF- and DDF-aided cooperative system employing the APA scheme is depicted versus $d_{m}$ in Figs. 9(a) and 10(a), respectively, in comparison to that of the system employing the simple equal power allocation. We simply assume that multiple activated cooperating MSs are located at the same distance from the source MS. Observe in Figs. 9(a) and 10(a) that significant performance improvements can be achieved by the APA scheme for both the DAF- and DDF-aided systems, when the cooperating user is situated closer to the BS than to the source MS. However, due to the difference between the relaying mechanisms employed by the two above-mentioned cooperative systems, it is interesting to observe that the BER trends seen in Fig. 9(a) are quite different from those emerging from Fig. 10(a). Specifically, Fig. 9(a) demonstrates that it is desirable to choose multiple cooperating MSs closer to the BS than to the source MS in a DAF-aided cooperative system, espcially when optimally sharing the power among the cooperating users. By contrast, Fig. 10(a) reveals that the cooperating MSs roaming in the vicinity of the source MS are preferred for a DDF-aided system in the interest of maintaining a better BER performance. Additionally, by comparing the results of Figs. 9(a) and 10(a), we found that the DDF-aided system suffers from a less dramatic performance loss than its DAFaided counterpart, when employing the equal-power allocation scheme, provided that the multiple cooperating MSs are closer to their desired locations. Hence, for the sake of reducing the complexity, the DDF-aided cooperative system may simply employ an equal-power allocation, while being still capable 


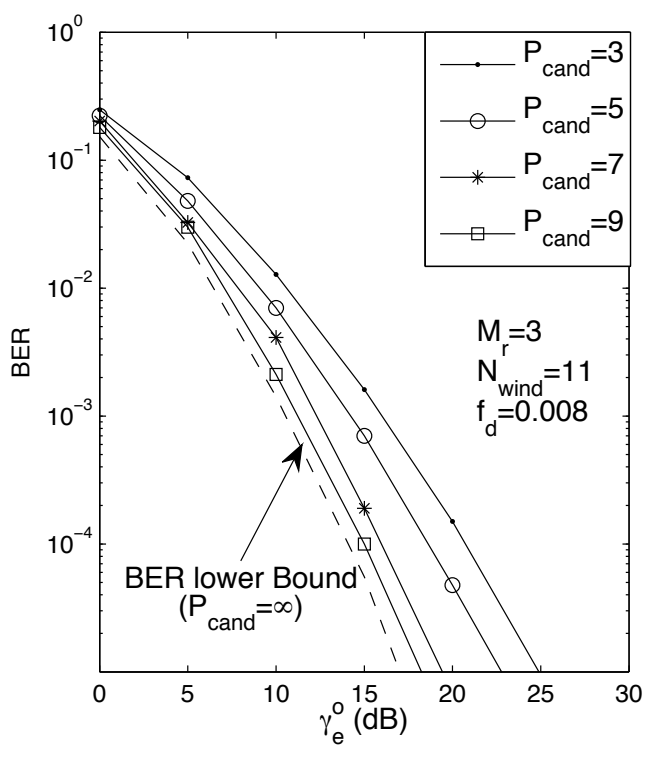

(a) BER performance

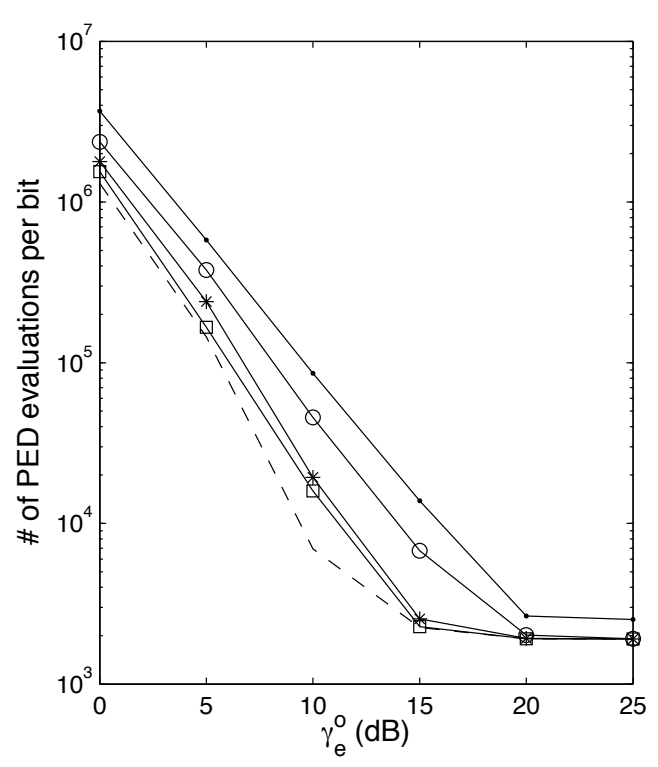

(b) Complexity imposed by the MSDSD

Fig. 8. The effects of the size of the cooperating RS pool on the DAF-aided DQPSK modulated user-cooperative cellular uplink employing the CUS and APA schemes in a Rayleigh fading channel associated with $f_{d}=0.008$ and $v=3$, where $M_{r}=3$ cooperating users are activated.

of achieving a desirable performance by solely invoking the CUS scheme.

In parallel, the BER performance of the DAF- and DDFaided systems is depicted against $P_{s} / P$ in Figs. 9(b) and 10(b), respectively. A significant performance gain can be attained by locating the cooperating MS at the optimum position, rather than in the middle of the source MS and BS path for both systems, which is expected to become even higher, as the number of actively cooperating MSs, $M_{r}$, increases. For optimum cooperating user location, instead of allocating the majority of the total transmit power to the source MS as it was suggested by Fig. 9(b) for the DAF-aided system

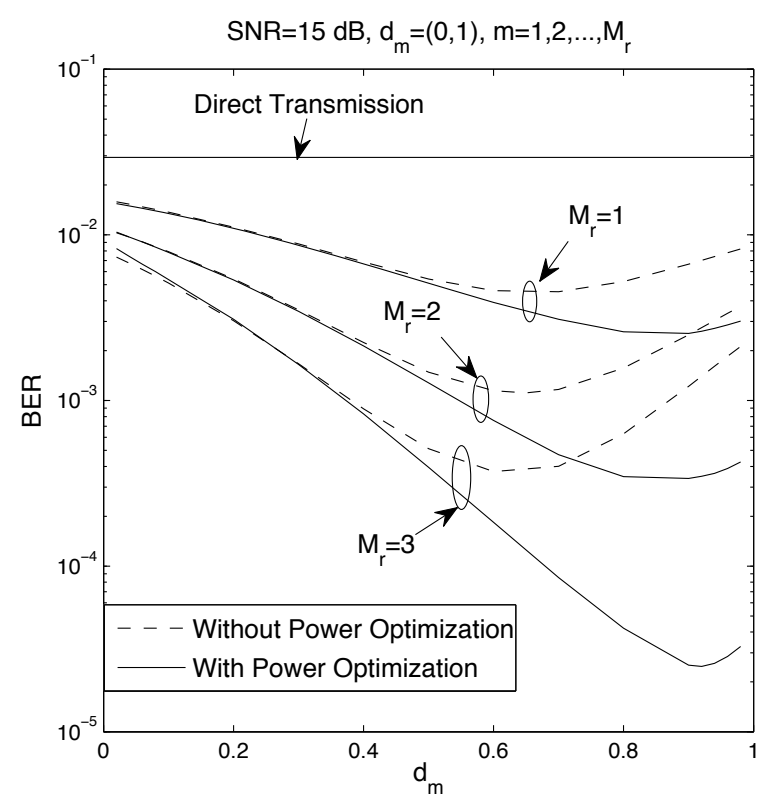

(a) Adaptive power allocation

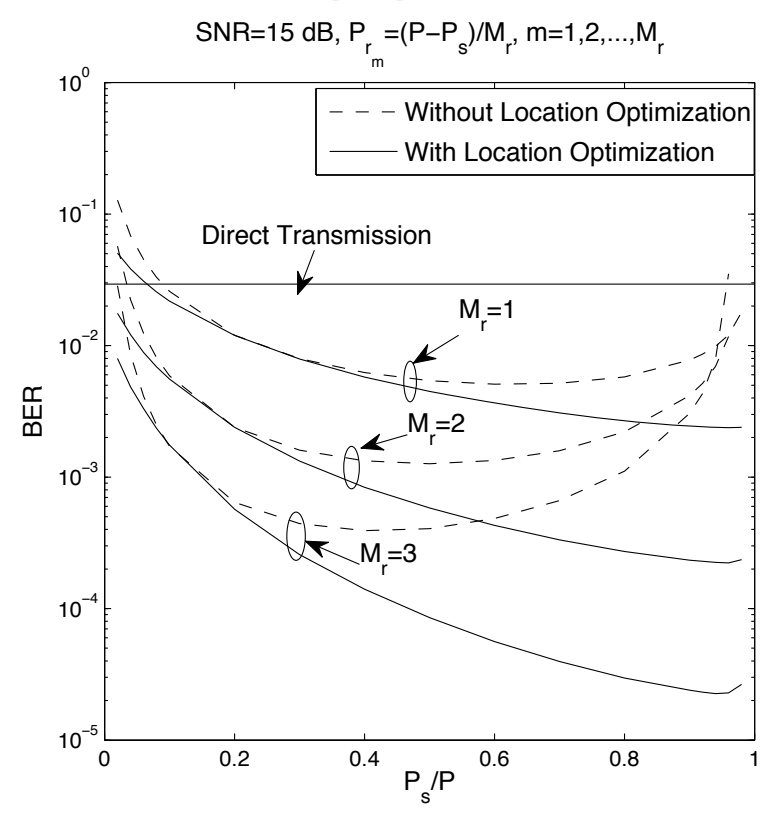

(b) Relay location optimization

Fig. 9. Power and relay location optimization for DQPSK modulated DAFaided cooperative cellular systems having $M_{r}$ activated RSs in a Rayleigh fading channel associated with $v=3$.

in the interest of achieving an improved BER performance - the results of Fig. 10(b) suggest that only about half of the total power has to be assigned to the source MS, if the DDF scheme is used. Furthermore, the information bit stream is CRC coded by the source MS for the DDF-aided system in order to carry out the CRC checking at the cooperating MS. Hence, for the sake of maintaining a relatively high effective throughput, two different transmission packet lengths are used, namely, $L_{p}=128$ and $L_{p}=64$ DQPSK symbols. However, a larger value of $L_{p}$ may result in a worse endto-end performance, since the activated RS is less likely to participate in signal forwarding owing to the increased 


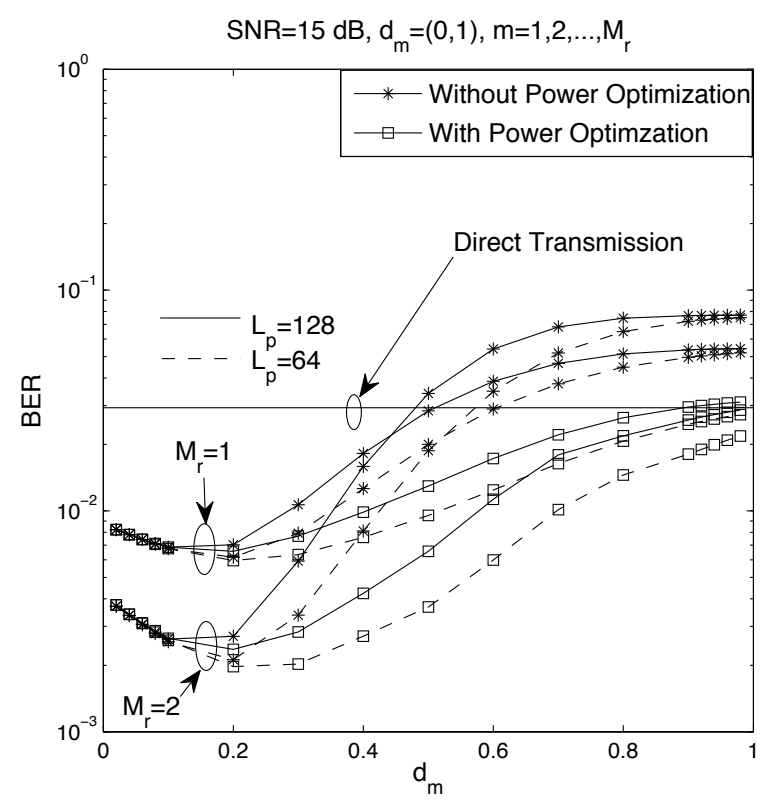

(a) Adaptive power allocation

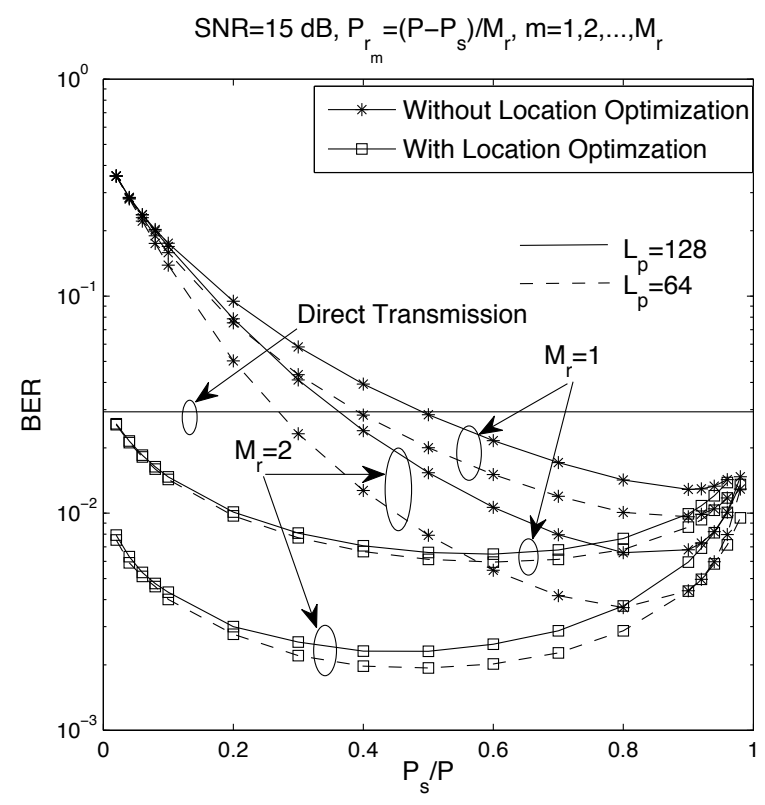

(b) Relay location optimization

Fig. 10. Power and RS location optimization for the DQPSK modulated DDF-aided cooperative cellular systems having $M_{r}$ activated RSs in a Rayleigh fading channel associated with $v=3$.

PLR. Fortunately, as observed in Figs. 10(a) and 10(b), the originally significant performance differences caused by the different packet lengths of $L_{p}=128$ and $L_{p}=64$, can be substantially reduced for the DDF-aided system, provided that the cooperating user is situated at or near the optimum location. Finally, the comparison of the DAF- and DDF-aided systems is summarized in Table II.

\section{Resource-Optimized Hybrid Cooperative Cellular Uplink}

In the light of the complementarity of the two relaying mechanisms as revealed by their comparison in Section IV-B, a more flexible cooperative scenario can be created in order
TABLE III

RESOURCE AlLocation FOR THE Hybid COOPERATIVE UPLINK

\begin{tabular}{|c|c|c|c|}
\hline$M_{r}$ & $P / N_{0}(\mathrm{~dB})$ & $P_{s}, P_{r_{1}}, P_{r_{M_{2}}}$ & {$\left[d_{1}, d_{2}\right]$} \\
\hline \multirow{3}{*}{2} & 10 & {$[0.702,0.202,0.096]$} & {$\left[\begin{array}{lll}0.26, & 0.86\end{array}\right]$} \\
\hline & 20 & {$[0.702,0.202,0.096$} & $0.31,0.86$ \\
\hline & $\overline{30}$ & $0.702,0.202,0.096$ & $0.31,0.91$ \\
\hline
\end{tabular}

to enhance the attainable performance, where the cooperating MSs roaming in different areas between the source MS and the BS may be activated and the relaying schemes employed by each activated RS may be adaptively selected, while maintaining a moderate complexity. For the sake of simplicity, let us now consider the hybrid cooperative cellular uplink as portrayed in Fig. 4, where $M_{r}=2$ cooperating MSs roaming in the preferred DDF- and DAF-RS-area are activated. The particular cooperative protocol employed by the activated RSs is determined according to the specific area, which they happen to be situated in. In order to capitalize on the complementarity of the DAF and DDF schemes, it may be assumed that one of the cooperating MSs is activated in the preferred area of the DAF-RS, while the other from the 'DDF-area', although naturally, there may be more than one cooperating MSs roaming within a specific desirable area. Then, under the assumption that the first selected cooperating MS is roaming in the 'DDF-area', while the second one is roaming in the 'DAF-area' of Fig. 4, the cooperative resource allocation is optimized as shown in Table III based on the minimum BER criterion. The derivation of the theoretical BER for the hybrid cooperative system may also follow the unified approach outlined in Section II-B. As expected, Table III reveals that the 'DDF-area' and the 'DAF-area' are still located in the vicinity of the source MS and the BS, respectively. Additionally, the majority of the total transmit power, i.e. about $70 \%$, should be allocated to the source MS, while $\frac{2}{3}$ of the remaining power should be assigned to the cooperating MS roaming in the 'DDF-area'.

The BER performance of our proposed hybrid cooperative cellular uplink, where $M_{r}=2$ out of $\mathcal{P}_{\text {cand }}=8$ cooperating MSs are activated, is portrayed in comparison to that of its DAF- and DDF-aided counterparts in Fig. 11. Remarkably, as demonstrated by Fig. 11, the hybrid cooperative system outperforms both the DAF- and DDF-aided systems, regardless whether the joint-CUS-APA scheme is activated or not. These conclusions remain valid across a wide SNR range of our interest, although the performance advantage of the hybrid scheme over the latter two systems decreases in the context of the joint-CUS-APA scheme. Furthermore, as the SNR increases, the DDF-aided system is expected to become superior to the other two systems, since it performs best, when error-free transmissions can be achieved between the source MS and the RS. By contrast, if the SNR is low, the DAF-aided system performs best amongst the three. In addition to the performance advantage of the joint-CUS-APA-aided hybrid cooperative system, the overall system complexity becomes more moderate in comparison to that of DDF-aided system, since only half of the activated MSs have to decode and re-encode the received signal prior to forwarding it. Therefore, the proposed hybrid cooperative system employing the 
TABLE II

COMPARISON BETWEEN THE DAF- AND DDF-AIDED COOPERATIVE CELLULAR UPLINKS

\begin{tabular}{|l|r|r|}
\hline & DAF-Aided Cooperative Uplink & DDF-Aided Cooperative Uplink \\
\hline Overall Performance & better when SR link quality is poor & better when SR link quality is good \\
\hline Complexity at RS & low (amplify) & high (decode and re-encode) \\
\hline Potential Drawbacks & noise amplification & error propagation \\
\hline $\begin{array}{l}\text { Performance's Sensitivity to } \\
\text { Source-Relay Link Quality }\end{array}$ & relatively moderate \\
\hline $\begin{array}{l}\text { Performance's Sensitivity } \\
\text { to Packet Length } L_{p}\end{array}$ & insensitive \\
\hline Desirable RS Locations & near the BS \\
\hline $\begin{array}{l}\text { Desirable Transmit Power } \\
\text { for the Source MS }\end{array}$ & about $88 \%$ of the total power & strong without CUS, minor with CUS \\
\hline $\begin{array}{l}\text { Worst Case Performance } \\
\text { (Bad Resource Allocation) }\end{array}$ & slightly better than the non-cooperative system & significantly worse than the non-cooperative system \\
\hline Importance of CUS and APA & equally important & CUS is significantly more important \\
\hline MSDSD Design Assumptions & Gaussian distributed received signal \& noise & error-free decoding at RS \\
\hline $\begin{array}{l}\text { Performance Gain Achieved } \\
\text { by MSDSD }\end{array}$ & channel-induced error-floor completely eliminated & channel-induced error-floor completely eliminated \\
\hline
\end{tabular}

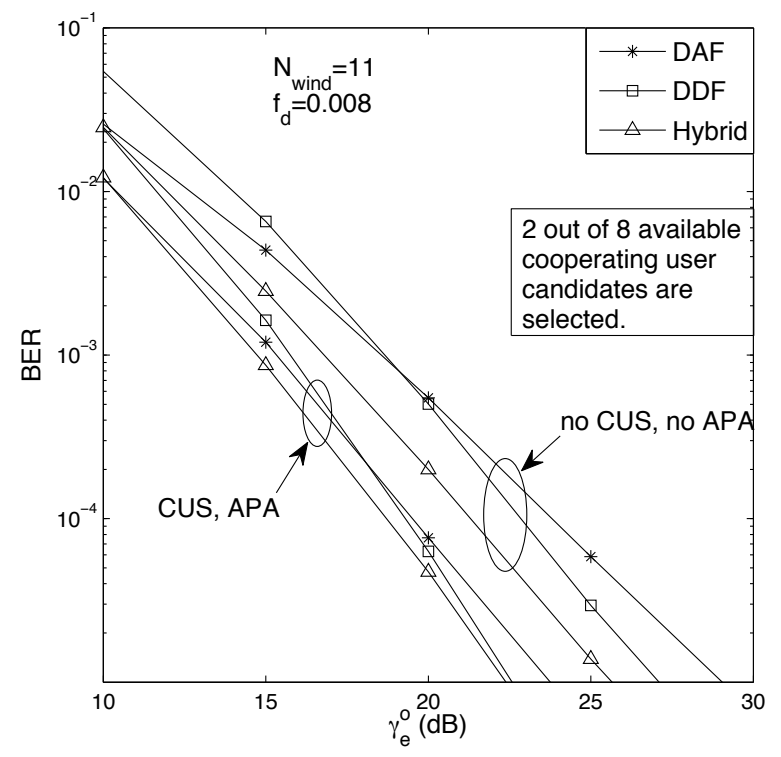

Fig. 11. Performance improvement by the joint CPS and CUS for the DQPSK modulated user-cooperative cellular uplink employing the MSDSD in a Rayleigh fading channel associated with $f_{d}=0.008$ and $v=3$, where 2 out of 8 cooperating user candidates are activated.

joint-CUS-APA scheme is capable of achieving an attractive performance, despite maintaining a moderate overall system complexity.

\section{Code-Rate-Optimized Cooperative System}

As seen in Section II, the cooperative system's performance is expected to be better than that of classic direct transmissions in the absence of channel coding. This is because the attainable transmit diveristy gains as well as path loss reductions achieved by the cooperative relay-aided system translate into substantially enhanced robustness against fading for a given transmit power, or into a significantly reduced transmit power requirement for the same BER performance $[48,49,52]$. The achievable cooperative performance may be further enhanced by beneficial power allocation and RS selections schemes using the minimum-BER criterion, as observed in Section IV. Employing other criteria, such as for example the minimum- outage-probability criterion used in [50], is also a feasible design alternative.

Moreover, the cooperative diversity gains promised by the cooperative system considered are actually achieved at the cost of suffering a significant so-called multiplexing loss compared to direct transmissions, which is imposed by the half-duplex communications of practical transceivers. Furthermore, the cooperative diversity gains achieved by the relayaided system over its direct-transmission based counterpart may become modest in practical channel coded scenarios, where the interleaving and channel coding gains dominate. Therefore, when a cooperative wireless communication system is designed to approach the maximum achievable spectral efficiency by taking the cooperation-induced multiplexing loss into account, it is not obvious, whether or not the relayaided system becomes superior to its direct-transmission based counterpart. In fact, recent research in [30] has revealed that the AF-based cooperative system suffers a significant capacity loss in comparison to the conventional direct transmission system.

Hence, in this section we will answer the fundamental question for the DDF-based cooperative system, whether it is worth introducing cooperative mechanisms into the development of wireless networks in the interest of achieving a high spectral efficiency from a pure capacity perspective. Moreover, in the open literature, the DDF-aided cooperative system has mainly been investigated in the context of fixed and predetermined time resource allocation (TRA) between the source MS and RS in TDMA scenarios. Thus, the optimization of TRA or equivalently the coding rates employed by the source MS and RS will also be discoursed in this section. For the sake of carrying out a fair comparison between the cooperative system and its direct-transmission counterpart as well as to put our emphasis on investigating the maximum achievable transmission rate, a single-relay-aided cooperative scenario is considered under the simplifying assumption of equal-power allocation $\left(P_{s}=P_{r}=P\right)$ and mid-point RS location in the channel-coded scenarios. 


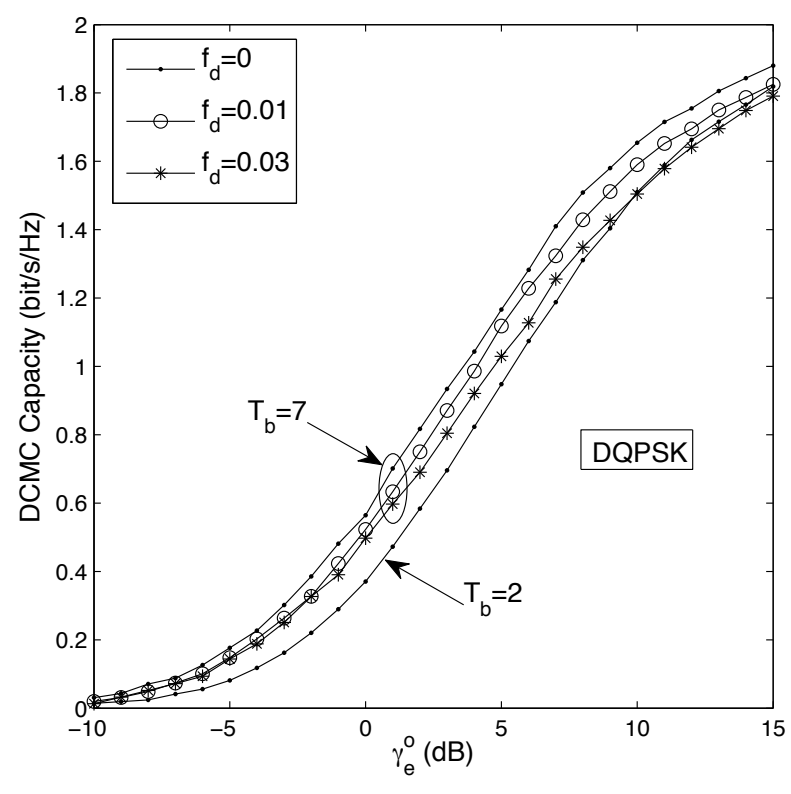

Fig. 12. The DCMC capacity of the differentially modulated point-topoint transmission associated with various normalized Doppler frequencies and fading block sizes $T_{b}$.

\section{A. Non-Coherent Channel Capacity for Point-to-Point Links}

If we assume that the Rayleigh fading block size over which the fading envelope is assumed to be correlated is $T_{b}$, then the $T_{b}$-symbol transmission model of the point-topoint (P2P) system may be obtained in the same form as (7) by omitting the relay-link-related elements in $\underline{\mathbf{y}}, \underline{\mathbf{S}}, \underline{\mathbf{h}}$ and $\underline{\mathbf{w}}$. Since each element of the $T_{b}$ consecutively transmitted symbols stored in the diagonal matrix $\underline{\mathbf{S}}$ 's diagonal vector $\mathbf{s}$ is chosen independently from an $M_{c}$-point constellation set $\mathcal{M}_{c}$ with equal probabilities, the non-coherent DCMC capacity can be expressed as a function of the SNR $\gamma$ as $C_{p 2 p}(\gamma)=I(\mathbf{s} ; \underline{\mathbf{y}})=H(\underline{\mathbf{y}})-H(\underline{\mathbf{y}} \mid \mathbf{s})$, where $I(\mathbf{a} ; \mathbf{b})$ represents the average mutual information (MI) per symbol between the channel input a and the corresponding channel output $\mathbf{b}$, while $H(\mathbf{x})$ represents the differential entropy [83] of a random vector $\mathbf{x}$. According to [83], $H(\mathbf{y} \mid \mathbf{s})$ may be readily calculated as $H(\mathbf{y} \mid \mathbf{s})=\log \operatorname{det}(\pi \mathrm{e} \Psi)$ bits. On the other hand, the entropy $\bar{H}(\mathbf{y})$ of the continuous-valued faded and noise-contaminated received signal vector $\mathbf{y}$ cannot be evaluated in a closed form. When the fading block size $T_{b}$ is limited, a practical approach to the numerical evaluation of $H(\underline{\mathbf{y}})=-\int p(\underline{\mathbf{y}}) \log p(\underline{\mathbf{y}}) \mathrm{d} \underline{\mathbf{y}}$ is to carry out Monte-Carlo integration as used in [84].

The non-coherent DCMC capacity of the time-selective block-fading channel using the DQPSK modulation scheme is plotted in Fig. 12 for various fading block sizes of $T_{b}=2$ and 7 as well as for various normalized Doppler frequencies of $f_{d}=0,0.01$ and 0.03 . Observe in Fig. 12 that although an identical differential modulation scheme is employed, the maximum achievable spectral efficiency is dependent on the fading block size $T_{b}$. On the other hand, according to [60], the predictability of the channel is characterized by the rank $Q$ of the channel's covariance matrix $\Sigma_{h}$. For example, the standard block-fading channel $\left(f_{d}=0\right)$, where the fading envelope remains constant over the entire fading block is associated with the most predictable fading envelope, when the channel's covariance matrix has a rank of $Q=1$. By contrast, the fading process has a finite differential entropy and becomes less predictable, when we have $Q=T_{b}$. Consequently, when we have an increased channel unpredictability owing to an increased Doppler frequency, a capacity loss is observed in Fig. 12. Hence, the non-coherent DCMC capacity of a time-seletive block-fading channel is dependent on both the fading block size $T_{b}$ and the fading correlation over blocks characterized by the channel covariance matrix $\Sigma_{h}$, as suggested by Fig. 12 .

\section{B. Capacity of the DDF-aided Cooperative System}

Based on the general upper and lower bounds of the capacity of half-duplex relay systems in [85] and on the fact that in our DDF-aided half-duplex relay system the source MS remains silent during Phase II, the single-relay-aided DDF cooperative system capacity can be written as:

$$
\begin{aligned}
& C_{\text {coop }}^{\operatorname{DDF}}\left(\gamma_{e}^{o}, \alpha\right) \\
& =\min \left\{\alpha I\left(\mathbf{s}_{s}^{I} ; \underline{\mathbf{y}}_{r}^{I}\right), \alpha I\left(\mathbf{s}_{s}^{I} ; \underline{\mathbf{y}}_{d}^{I}\right)+(1-\alpha) I\left(\mathbf{s}_{r}^{I I} ; \underline{\mathbf{y}}_{d}^{I I}\right)\right\},
\end{aligned}
$$

where $I\left(\mathbf{s}_{s}^{I} ; \underline{\mathbf{y}}_{d}^{I}\right), I\left(\mathbf{s}_{r}^{I I} ; \underline{\mathbf{y}}_{d}^{I I}\right)$ and $I\left(\mathbf{s}_{s}^{I} ; \underline{\mathbf{y}}_{r}^{I}\right)$ are the constrained information rates of the $\overline{\mathrm{S}}-\mathrm{D}, \mathrm{R}-\mathrm{D}$ and $\overline{\mathrm{S}}$-R links, respectively. Moreover, the TRA-factor $\alpha$ is defined as $\alpha \triangleq \frac{L_{s}}{L_{s}+L_{r}}=$ $\frac{R_{r}}{R_{s}+R_{r}}$, since the ratio of the time durations $\left(L_{s}, L_{r}\right)$ used by the source and RS is inversely proportional to the ratio of the channel code rates $\left(R_{s}, R_{r}\right)$ employed by them. Hence, given the target transmission rate, the question arises as to how to design the cooperative system by optimizing the TRA or equivalently optimizing the code rate allocation between the source and RS in the interest of requiring the globally minimum overall equivalent SNR, i.e. $\gamma_{e}^{o}$, which has to be lower than the minimum $\gamma_{e}^{o}$ required by its direct-transmission based counterpart in order to make the cooperative system design meaningful. This challenging issue will be addressed in the rest of this treatise.

\section{Optimum Code Rate for the Cooperative System}

Since the first term within the 'min' function of the abovementioned DDF system's capacity expression of (10) increases with $\alpha$, while the second term decreases with the TRAfactor $\alpha$, the optimum value of $\alpha$, which maximizes the achievable information rate of the single-relay-aided DDFbased cooperative system, is the point obtained by equating the two arguments of the 'min' function, which gives rise to:

$$
\hat{\alpha}^{\text {opt }}\left(\gamma_{e}^{o}\right)=\frac{I\left(\mathbf{s}_{r}^{I I} ; \underline{\mathbf{y}}_{d}^{I I}\right)}{I\left(\mathbf{s}_{s}^{I} ; \underline{\mathbf{y}}_{r}^{I}\right)-I\left(\mathbf{s}_{s}^{I} ; \underline{\mathbf{y}}_{d}^{I}\right)+I\left(\mathbf{s}_{r}^{I I} ; \underline{\mathbf{y}}_{d}^{I I}\right)} .
$$

On the other hand, given a fading block size $T_{b}$ and a $M_{c^{-}}$ ary DPSK scheme, the actual transmission rate of the singlerelay-aided DDF cooperative system is a function of both $R_{s}$ and $\alpha$, which may be expressed as:

$$
R_{\text {coop }}^{\mathrm{DDF}}\left(R_{s}, \alpha\right)=\alpha R_{s} \log _{2} M_{c} .
$$




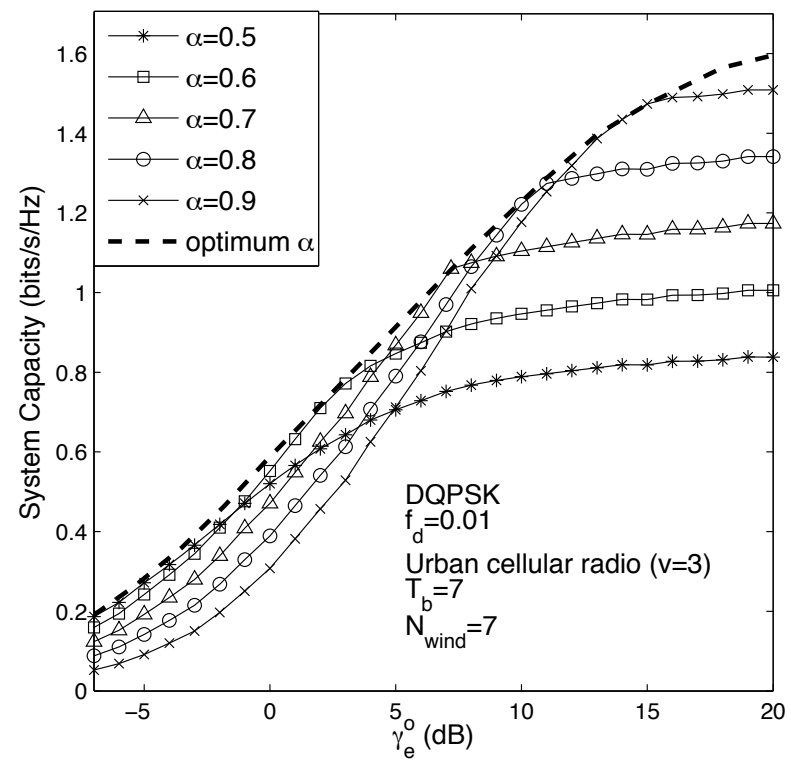

Fig. 13. Capacity of the single-relay-aided DDF cooperative system.

Hence, based on the aforementioned DDF system's capacity in (10) and actual-transmission-rate expression of (12), the optimum code rate pair $\left\{\hat{R}_{s}^{o p t}, \hat{R}_{r}^{\text {opt }}\right\}$ employed by the source and RS associated with the optimum TRA-factor of (11) may be expressed using a few mathematical manipulations as:

$$
\begin{aligned}
\hat{R}_{s}^{o p t} & =\frac{I\left(\mathbf{s}_{s}^{I} ; \underline{\mathbf{y}}_{r}^{I}\right)}{\log _{2} M_{c}} ; \\
\hat{R}_{r}^{o p t} & =\frac{I\left(\mathbf{s}_{s}^{I} ; \underline{\mathbf{y}}_{r}^{I}\right) I\left(\mathbf{s}_{r}^{I I} ; \underline{\mathbf{y}}_{d}^{I I}\right)}{\left[I\left(\mathbf{s}_{s}^{I} ; \underline{\mathbf{y}}_{r}^{I}\right)-I\left(\mathbf{s}_{s}^{I} ; \underline{\mathbf{y}}_{d}^{I}\right)\right] \log _{2} M_{c}} .
\end{aligned}
$$

In Fig. 13 the single-relay-aided cooperative system's DCMC capacity associated with different values of $\alpha$ is depicted versus $\gamma_{e}^{o}$ in comparison to that of its code-rateoptimized (CRO) counterpart in conjunction with the optimum TRA-factor $\alpha$ of (11) in a typical urban cellular scenario [15]. As observed in Fig. 13, the system capacity is indeed maximized by optimizing the code-rates of the source and relay MSs.

\section{Capacity Comparison and Discussions}

In order to gain an insight into the benefits of the singlerelay-assisted DDF cooperative system over its conventional direct-transmission based counterpart from a pure capacity perspective, the DCMC capacity of the CRO cooperative system is depicted in comparison to that of the direct-transmission based one in Fig. 14. It may be observed in the figure that if the overall equivalent SNR is relatively low, the CRO DDF cooperative system exhibits a significantly higher capacity than its direct-transmission based counterpart in typical urban cellular scenarios. More specifically, in a shadowed urban area associated with $v=4$, the DDF system only requires one sixth of the total transmit power necessitated by its directtransmission based counterpart at a spectral efficiency of $0.5 \mathrm{bits} / \mathrm{s} / \mathrm{Hz}$. However, the achievable capacity gain may be

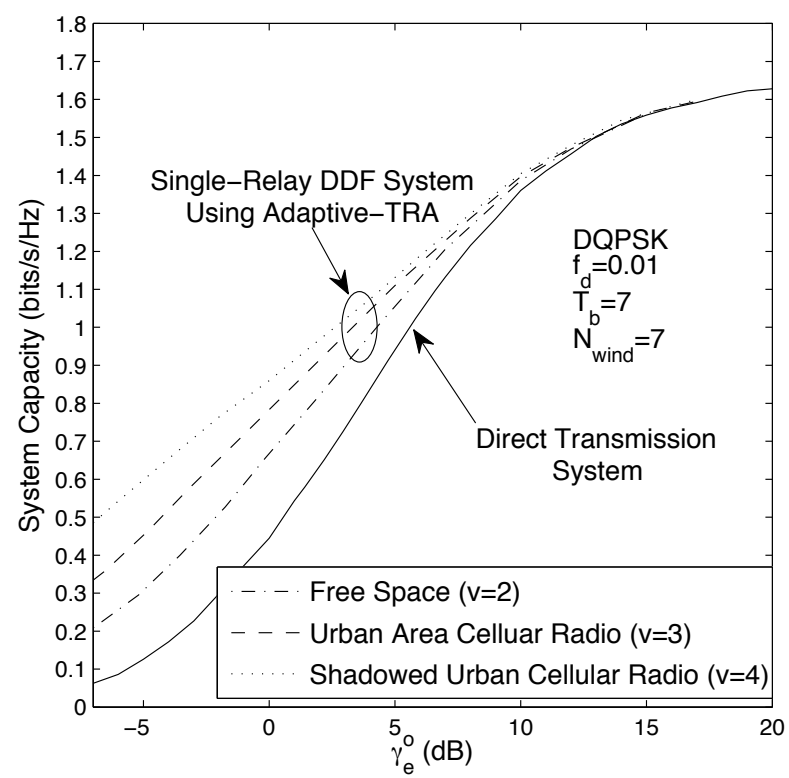

Fig. 14. Capacity comparison of the single-relay-aided cooperative system and its direct-transmission based counterpart.

substantially reduced, if we encounter a free-space propagation scenario associated with $v=2$, owing to the reduced pathloss-related power-gain achieved in the cooperative system. Moreover, as the overall equivalent SNR increases to a relatively high value, the benefits of invoking a single-relay-aided cooperative system for achieving a high spectral efficiency also become negligible.

\section{Code-Rate-Optimized Near-Capacity Design FOR THE DDF-AIDED COOPERATIVE SYSTEM}

In this section, a practical CRO near-capacity design framwork will be presented for the DDF-aided cooperative system in order to verify the theoretical benefits of employing the DDF scheme, as observed in Section V-D from a realistic system implementation perspective.

\section{A. Transceiver Design}

1) Capacity-Achieving Soft-Input Soft-Output MSDSD: In pursuit of a near-capacity performance for the channelcoded DDF-aided system, it is beneficial that the MSDSD at the turbo receiver is capable of incorporating/providing bitreliability-based soft information from/to the channel decoder. This is because the channel decoder benefits from exploiting the reliability information provided by the MSDSD and returns its improved-confidence soft-information to the MSDSD in the interest of iteratively increasing the resultant $a$ posteriori probability (APP), which is conventionally expressed in terms of the log-likelihood ratio (LLR) as $L_{D}\left(x_{k} \mid \underline{\mathbf{y}}\right)=\ln \frac{P\left[x_{k}=+1 \mid \mathbf{y}\right]}{P\left[x_{k}=-1 \mid \mathbf{y}\right]}$, where $x_{k}$ is the $k$ th element of the multiple-symbol-based transmitted bit vector $\mathbf{x}$ associated with the multiple-symbolbased transmitted symbol vector $\mathbf{s}$. Hence, a soft-input softoutput (SISO) MSDSD was devised for the iterative detection assisted receivers in [80], which was also demonstrated to be the capacity-achieving non-coherent detection scheme. 


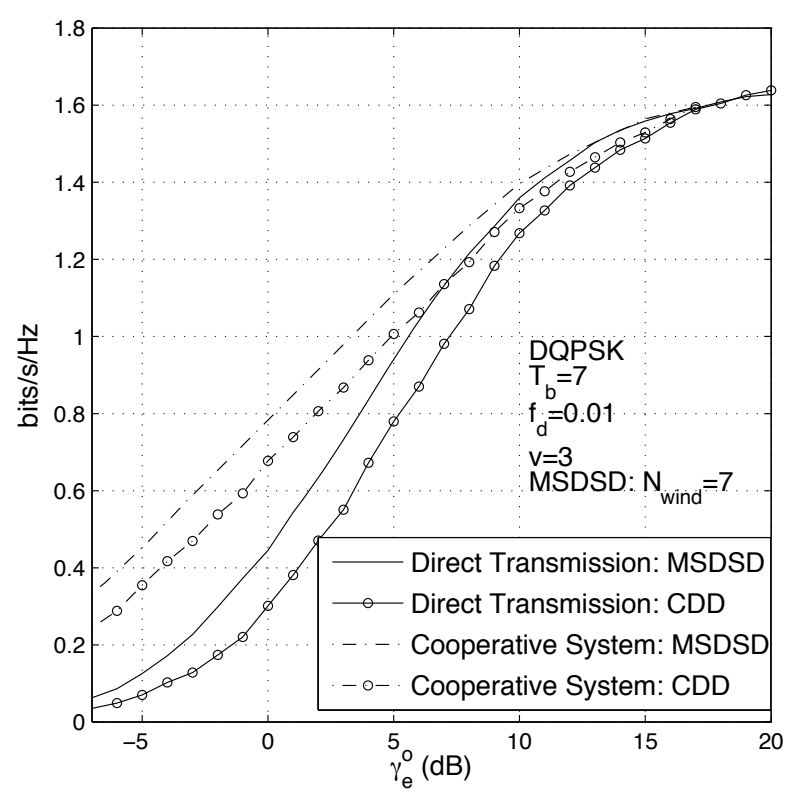

Fig. 15. Maximum achievable rate of the CDD- and MSDSD-aided systems for both direct transmission and the relay-aided transmission.

The maximum achievable rate of the cooperative system and its direct-transmission based counterpart are plotted against the overall equivalent SNR $\gamma_{e}^{o}$ in Fig. 15 for both the CDD- and MSDSD-aided scenarios. Observe in Fig. 15 that within the SNR range of interest, an approximately $0.15-0.2$ bits $/ \mathrm{s} / \mathrm{Hz}$ higher 'near-error-free' transmission rate can be supported with the aid of the MSDSD in comparison to the CDDaided scheme for both the direct transmission and cooperative systems, when the fading block size $N_{\text {wind }}=T_{b}=7$. Hence, the MSDSD is advocated in our ensuing high-spectralefficiency cooperative system design.

2) Irregular Distributed Differential Encoding/Decoding: The transceiver architecture proposed for the single-relayaided DDF cooperative system is portrayed in Fig. 16. At the transmitter of the source MS of Fig. 16, we use a conventional differential modulation scheme, such as DQPSK, which is amalgamated with the unity-rate-code (URC) encoder in order to create a two-stage inner code ${ }^{2}$, whereas an Irregular Convolutional Code $^{3}$ (IrCC) associated with an average code rate of $R_{s}$, namely $\operatorname{IrCC}_{s}$, is employed as the outer code

\footnotetext{
${ }^{2}$ The benefits of the URC is that it has an infinite impulse response (IIR) and hence efficiently spreads the extrinsic information amongst the decoder components. We use here the two-stage 'inner' code terminology to indicate that the demodulator and the URC decoder exchange their extrinsic information as many times as needed, until no more MI improvement is achievable and hence they may be considered as an amalgamated block

${ }^{3}$ The philosophy of Irregular Convolutional Code was proposed in [86] for approaching the achievable system capacity. As detailed in [87], their design is based on EXtrinsic Information Transfer (EXIT) charts [88] by exploiting the property that a concatenated coding scheme is capable of exhibiting an infinitesimally low BER at near-capacity SNRs, if the area between the EXITcurves of the inner- and outer-codes is vanishingly low. This condition may be satisfied by matching the outer IrCC decoder's EXIT-curve to that of the inner decoder's curve. More specifically, a dynamic programming procedure is used to carry out this MI curve-matching by appropriately encoding certain fractions of the input bit-stream with the aid of different-rate $\mathrm{CCs}$, where the fractions are the result of the EXIT-tunnel area minimization, again, as detailed in [86].
}

for the sake of achieving a near-error-free transmission at SNRs close to the capacity of the SR link. The corresponding URC decoder assisted three-stage receiver proposed for the relay is also portrayed in Fig. 16. Specifically, at the receiver of the relay, which is constituted by three modules, namely the $\mathrm{MSDSD}_{s}$, the $\mathrm{URC}_{s}$ decoder and the $\mathrm{IrCC}_{s}$ decoder, extrinsic information is exchanged amongst the modules in a number of consecutive iterations. As shown in Fig. 16, $A(\cdot)$ represents the a priori information expressed in terms of LLRs, while $E(\cdot)$ denotes the corresponding extrinsic information. Again, the basic idea behind the implementation of the three-stage concantenated transceiver at the source MS and RS is to improve the convergence behavior of the iterative detection based system with the aid of the URC, which will be demonstrated in Section VI-B2. At the two-stage ${ }^{4}$ serially concatenated transmitter of the relay in Fig. 16, the estimated data bit stream is fed through the interleaver $\pi_{r 1}$ prior to the $\mathrm{IrCC}_{r}$ encoder having an average code rate of $R_{r}$, in order to construct a distributed turbo code [26] together with the source MS, resulting in an Irregular Distributed Differential (IrDD) coding scheme, under the assumption of error-free decoding at the RS.

According to the principles of the distributed turbo decoding mechanism proposed in [26], the novel iterative receiver of the destination BS seen in Fig. 16 is used for decoding the IrDD coded stream jointly created by the source and relay MSs. To be specific, the first part of the iterative receiver is an amalgamated "MSDSD ${ }_{s}-\mathrm{URC}_{s}-\mathrm{IrCC}_{s}$ " iterative decoder, which is used to iteratively decode the signal directly received from the source during phase I, while the second part is constituted of the $\mathrm{MSDSD}_{r}$ detector and the $\mathrm{IrCC}_{r}$ decoder, which is employed to iteratively decode the signal forwarded by the RS during phase II. Furthermore, since the "MSDSD ${ }_{s}$ $\mathrm{URC}_{s}-\mathrm{IrCC}_{s}$ " decoder and the "MSDSD $r-\mathrm{IrCC}_{r}$ " decoder may be regarded as the two component decoders of a turbo receiver, the extrinsic information exchange between them, which is referred to as the "outer iteration", is expected to significantly enhance the achievable coding gain. Consequently, in comparison to the conventional relay-aided cooperative system, where a simple repetition code is constructed, the extra coding gain achieved by the proposed IrDD coding scheme may be interpreted as the joint benefit of the interleaving gain of the turbo code and the turbo processing gain of the outer iterations. The transceiver design rationale for the DDF-aided system is summarized in the first part of Table IV.

\section{B. Code-Rate-Optimized Near-Capacity Code Design}

In this section, we propose a practical near-capacity design framework, which enables the proposed $\mathrm{IrDD}$ scheme to approach the cooperative system's capacity. Both the $\mathrm{IrCC}_{s}$ and $\mathrm{IrCC}_{r}$ will be optimized based on a set of 17 subcodes associated with different coding rates ranging from 0.1 to

\footnotetext{
${ }^{4}$ Observe in Fig. 16 that the RS's receiver mirrors the three-stage architecture of the source's transmitter, but the RS's transmitter was simplified to a two-stage architecture. The rationale of this is that the SR link's decoding errors would result into avalanche-like error propagation, which justifies the powerful three-stage decoder architecture. By contrast, it may be deemed adequate to use a near-capacity two-stage architecture for the less critical RD link
} 


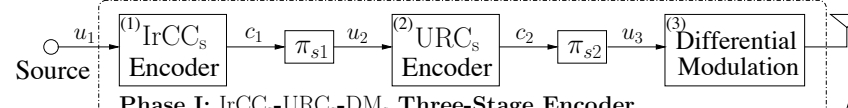

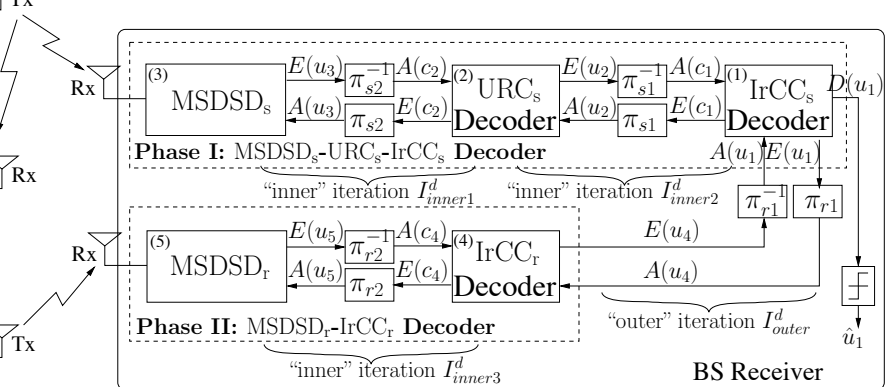

Phase I: MSDSD ${ }_{\mathrm{s}}-\mathrm{URC}_{\mathrm{s}}-\mathrm{IrCC}_{\mathrm{s}}$ Three-Stage Decoder

$\left.{ }^{(1)} \mathrm{IrCC}_{\mathrm{s}} \frac{A\left(c_{1}\right)}{\pi_{s 1}^{-1}} \frac{E\left(u_{2}\right)}{(2)} \mathrm{URC}_{\mathrm{s}} \frac{A\left(c_{2}\right)}{E\left(c_{2}\right)} \pi_{s 2}^{-1} \frac{E\left(u_{3}\right)}{A\left(u_{3}\right)}\right)^{(3)} \mathrm{MSDSD}_{\mathrm{s}} \quad \mathrm{Rx}_{\mathrm{Rx}}$

Relay

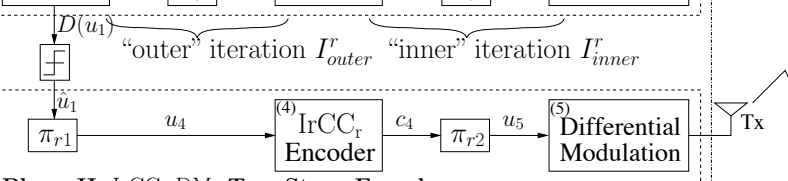

Phase II: $\mathrm{IrCC}_{\mathrm{r}}-\mathrm{DM}_{\mathrm{r}}$ Two-Stage Encoder Modulation

Relay Transceiver

Fig. 16. Schematic of the irregular distributed differential coding encoder/decoder.

TABLE IV

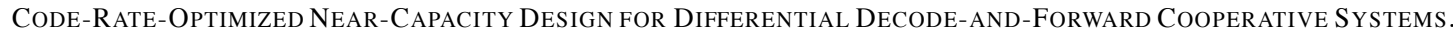

\begin{tabular}{|c|c|c|}
\hline \multirow[t]{2}{*}{$\begin{array}{l}\text { Transceiver } \\
\text { Design } \\
\text { Rationale }\end{array}$} & $\begin{array}{l}\text { Ir-DD } \\
\text { coding } \\
\text { (source+relay, } \\
\text { Fig.16) }\end{array}$ & $\begin{array}{l}\text { "MSDSD } \text {-URC }_{s} \text { " decoder at the RS capable of reaching the }(1.0,1.0) \text { point of the EXIT ch } \\
\text { Relay's receiver: URC-aided 3-stage receiver employing the MSDSD. Significantly enhanced i } \\
\text { be achieved by the MSDSD in comparison to the CDD. (see Fig. 17) } \\
\text { Relay's transmitter: 2-stage receiver employing } \operatorname{IrCC}_{r} \text { having a code rate of } R_{r} \text {, an interleav }\end{array}$ \\
\hline & $\begin{array}{l}\text { estinat } \\
\text { g.16) }\end{array}$ & $\begin{array}{l}\text { Destination's receiver: } \\
\text { 1). Constituted of two parts: first part is a three-stage receiver identical to relay's receiver iterative decoding } \\
\text { the signal received in broadcast phase; second part is a two-stage receiver corresponding to relay's transmitter, } \\
\text { iterative decoding the signal received in relay phase. } \\
\text { 2). Extrinsic information exchanges between the first and second parts. (see Fig. 18) }\end{array}$ \\
\hline Near-Capacity & \multicolumn{2}{|c|}{ 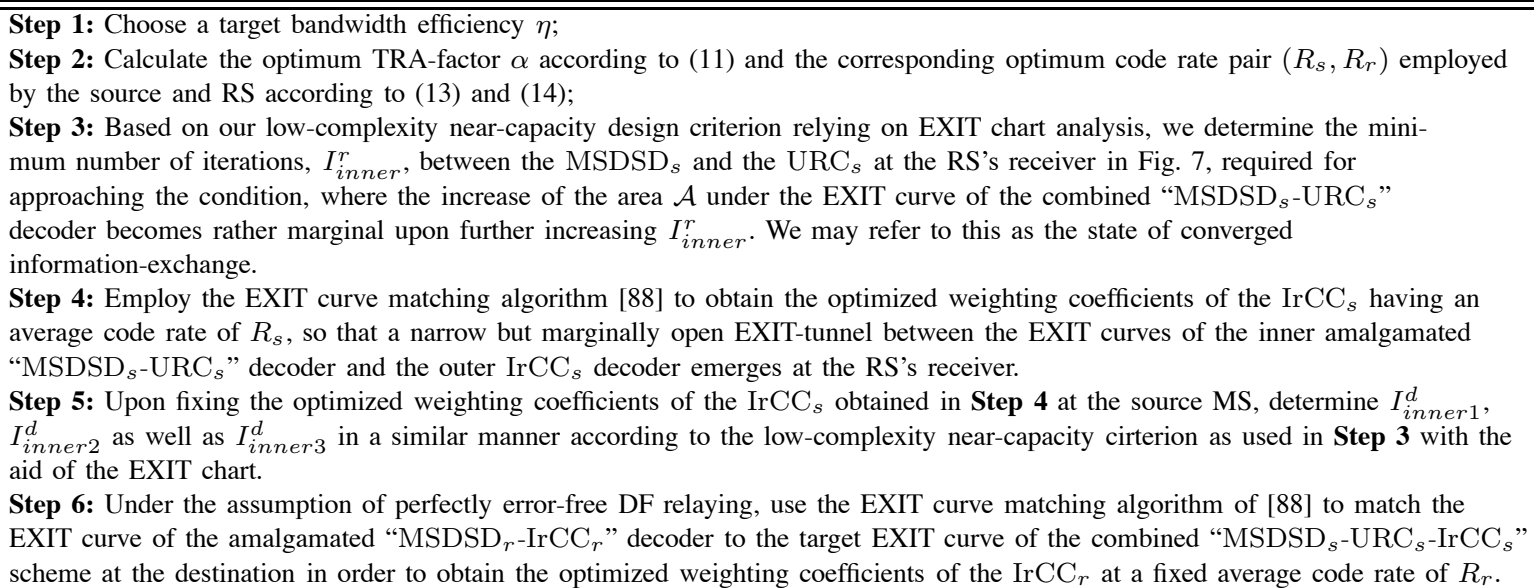 } \\
\hline
\end{tabular}

0.9. Two scenarios, namely, the typical urban cellular radio Scenario I and the free-space Scenario II are considered in a time-selective block Rayleigh fading propagation environment. Without loss of generality, the target bandwidth efficiency is chosen to be $\eta=0.5 \mathrm{bits} / \mathrm{s} / \mathrm{Hz}$ and DQPSK modulation scheme is employed. The optimum TRA-factor $\alpha$ and its corresponding optimum code rate pair $\left(R_{s}, R_{r}\right)$ employed by the source and RS can be obtained according to (11)(14) in the interest of maximizing the network's capacity, which are summarized in Table $\mathrm{V}$ together with all the other simulation parameters. The proposed CRO joint source-andrelay mode design procedure, which can be decoupled into two Extrinsic Information Transfer (EXIT) [88-91] curve matching problems under the assumption that the a priori
LLRs obey a symmetric Guassian distribution, is summarized in the second part of Table IV. Here we only detail the CRO near-capacity system design principles for Scenario I of Table V, while those of Scenario II are similar, but they are omitted for the reasons of space economy.

1) A Brief Review of the EXIT Chart and its Properties: The concept of EXIT charts was proposed by ten Brink in [90] as an efficient and powerful design tool for predicting and analyzing the convergence behavior of iterative decoding aided systems. Their capability of finding the decoding convergence threshold of the constituent codes may be exploited in the IrCC-aided near-capacity design to be highlighted in the ensuing sections. Their main features are outlined as follows:

- A Gaussian distribution exhibited by the LLR values is 
TABLE V

TRA-OPTIMIZED NEAR-CAPACITY SYSTEM DESIGN PARAMETERS.

\begin{tabular}{|c|c|c|}
\hline & $\begin{array}{l}\text { Scenario I } \\
\quad(v=3)\end{array}$ & $\begin{array}{r}\text { Scenario II } \\
(v=2)\end{array}$ \\
\hline Fading Block Size $T_{b}$ & & 7 \\
\hline Normalized Doppler Frequency $f_{d}$ & & 0.01 \\
\hline Target Bandwidth Efficiency $\eta$ & & $0.5 \mathrm{bits} / \mathrm{s} / \mathrm{Hz}$ \\
\hline Modulation & & DQPSK \\
\hline Detector & & MSDSD \\
\hline Channel Code & 17 -subco & e-based IrCC \\
\hline Code Block Length (Source MS) & & 40960 \\
\hline $\begin{array}{l}\text { Theoretically Minimum Required } \gamma_{e}^{o} \\
\text { (refer to Fig. 14) }\end{array}$ & $-4.3 \mathrm{~dB}$ & $-2.1 \mathrm{~dB}$ \\
\hline $\begin{array}{l}\text { Optimum TRA-factor } \alpha^{o p t} \\
\text { (according to }(11) \text { ) }\end{array}$ & 0.56 & $\overline{0.61}$ \\
\hline $\begin{array}{l}\text { Optimum Average Code Rate } R_{s} \\
\text { (according to (13)) }\end{array}$ & 0.52 & 0.48 \\
\hline $\begin{array}{l}\text { Optimum Average Code Rate } R_{r} \\
\text { (according to (14)) }\end{array}$ & 0.66 & 0.75 \\
\hline
\end{tabular}

the prerequisite for accurately analyzing/predicting the iterative decoding convergence behavior with the aid of EXIT charts.

- The EXIT curve of a SISO constituent decoder may be obtained by modelling the a priori LLRs and computing the corresponding MI between the hard-decision based bits and the extrinsic LLRs.

- An open tunnel having no intersections between the EXIT curves of the inner and outer codes implies that a vanishingly low BER may be achieved, since the Monte-Carlo simulation based stair-case shaped decoding trajectory can reach the $(1,1)$ point of convergence in the EXIT chart upon traversing through the open tunnel by employing a sufficiently high number of iterations.

- The EXIT charts allow us to employ a low-complexity near-capacity criterion by predicting a slightly 'higherthan-necessary' SNR value, where the system is capable of achieving a near-error-free transmission, while avoiding a high number of iterations, which imposes an excessive complexity.

- Since the area, $\mathcal{A}$, under the inner code's EXIT curve indicates the maximum code rate allowed to be employed by the outer code for a near-error-free iterative decoding, the system's maximum achievable transmission rate can be readily computed.

2) Reduced-Complexity Near-Capacity Design at the Relay in Scenario I: Although it is not explicitly demonstrated here owing to the lack of space, the iterative information exchange between the $\mathrm{MSDSD}_{s}$ and $\mathrm{URC}_{s}$ blocks of the relay's receiver seen in Fig. 16 approaches convergence as early as the second iteration. Hence, the number of iterations between the $\mathrm{MSDSD}_{s}$ and $\mathrm{URC}_{s}$ blocks is set to $I_{\text {inner }}^{r}=2$, when we obtain the EXIT curve of the combined "URC ${ }_{s}$ MSDSD " inner decoder of the RS, as shown in Fig. 17. As indicated by the wide gap between the inner decoder's EXIT curves associated with $N_{\text {wind }}=2$ and $N_{\text {wind }}=7$ in Fig. 17, significantly enhanced iterative gains may be achieved in comparison to the CDD assisted system using $N_{\text {wind }}=2$, when jointly and differentially detecting $N_{\text {wind }}=7$ number of data symbols using the MSDSD. Furthermore, observe in Fig. 17 that when the URC is employed, which again has an

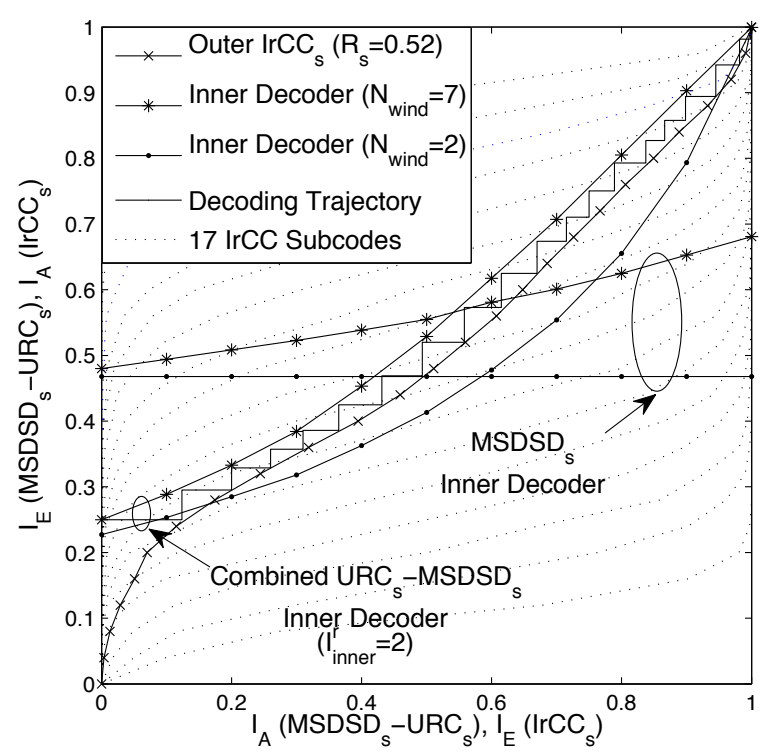

Fig. 17. EXIT curves of the MSDSD for various values of $N_{\text {wind }}$ (DQPSK, $\left.\gamma_{e}^{o}=0.7 \mathrm{~dB}, v=3, f_{d}=0.01\right)$.

infinite impulse response (IIR) due to its recursive encoder architecture, the EXIT curve of the URC-aided inner decoder, which has a lower starting point at $I_{A}=0$, is capable of reaching the $(1.0,1.0)$ point of perfect convergence to an infinitesimally low BER in the EXIT chart. Thus, the resultant steeper slope for the EXIT curve implies a reduced error floor and a higher 'turbo-cliff' SNR, above which decoding convergence to a vanishingly low BER becomes possible at the RS.

In practice, for the sake of avoiding a potentially excessive complexity at the RS, while approaching the capacity, a 'higher-than-necessary' EXIT curve associated with $N_{\text {wind }}=$ 7 may be ensured for the combined inner " $\mathrm{MSDSD}_{s}-\mathrm{URC}_{s}$ " decoder by having a slightly 'higher-than-necessary' overall equivalent SNR, for example, $\gamma_{e}^{o}=0.7 \mathrm{~dB}$, as depicted in Fig. 17. Then, using the EXIT curve of the amalgamated inner "MSDSD ${ }_{s}-\mathrm{URC}_{s}$ " decoder, the optimized weighting coefficients of the $\mathrm{IrCC}_{s}$ associated with the optimum code rate $R_{s}=0.52$ can be obtained with the aid of the EXIT curve matching algorithm of [88], resulting in a narrow but marginally open tunnel between the EXIT curves of the amalgamated "MSDSD ${ }_{s}-\mathrm{URC}_{s}$ " decoder and the $\mathrm{IrCC}_{s}$ decoder, as seen in Fig. 17.

3) Reduced-Complexity Near-Capacity Design at the Destination in Scenario I: Let us now consider the destination BS and optimize the weighting coefficients of the other IrCC, i.e. those of the $\mathrm{IrCC}_{r}$, employed by the RS's transmitter in Fig. 16. First of all, at the BS's receiver of Fig. 16, the desirable number of iterations, $I_{\text {inner } 1}^{d}$, between the $\mathrm{MSDSD}_{s}$ and the $\mathrm{URC}_{s}$ as well as that needed between the combined "MSDSD $s-\mathrm{URC}_{s}$ " decoder and the $\mathrm{IrCC}_{s}$ decoder, namely $I_{\text {inner2 }}^{d}$, have to be determined by examining the corresponding EXIT curves of the amalgamated "MSDSD ${ }_{s}-\mathrm{URC}_{s}-\mathrm{IrCC}_{s}$ " decoder associated with different values of $I_{\text {inner } 1}^{d}$ and $I_{\text {inner2 } 2}^{d}$. Based on our low-complexity near-capacity criterion, we set 


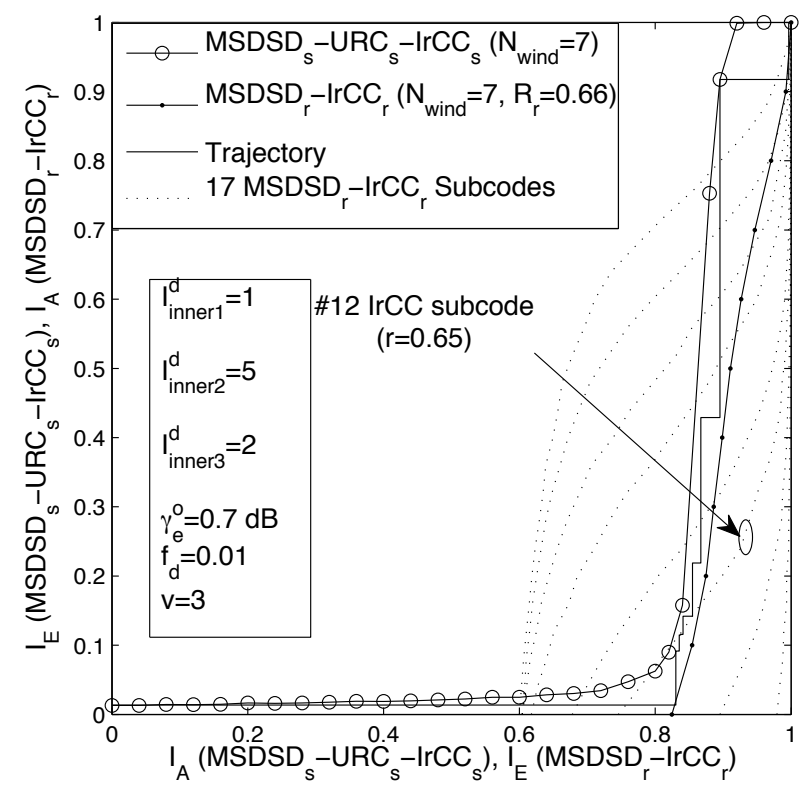

Fig. 18. Iterative decoding trajectory at the BS.

$I_{\text {inner1 }}^{d}=1$ and $I_{\text {inner } 2}^{d}=5$, since any further increase of the area under the EXIT curve of the combined "MSDSD ${ }_{s}$ $\mathrm{URC}_{s}-\mathrm{IrCC}_{s}$ " decoder in the interest of creating a wider EXIT tunnel is becoming rather marginal, when the number of iterations exceeds $I_{\text {inner } 1}^{d}=1$ and $I_{\text {inner } 2}^{d}=5$. Similarly, the desirable number of iterations between the $\mathrm{MSDSD}_{r}$ and the $\mathrm{URC}_{r}$ arrangements is chosen to be $I_{\text {inner3 }}^{d}=2$ by examining the EXIT chart of the combined "MSDSD ${ }_{r}$ $\mathrm{IrCC}_{r}$ " decoder. In Fig. 18 the resultant EXIT curve of the combined "MSDSD ${ }_{s}-\mathrm{URC}_{s}-\mathrm{IrCC}_{s}$ " decoder and those of the amalgamated "MSDSD ${ }_{r}$ - $\mathrm{IrCC}_{r}$ " subcodes are depicted.

Finally, upon fixing the optimum code rate of $R_{r}=0.66 \mathrm{ob}$ tained in Table V, we use the EXIT curve matching algorithm of [88] - which was detailed in [87] - to match the SNRdependent EXIT curve of the combined "MSDSD $r-\mathrm{IrCC}_{r}$ " decoder employed at the BS to the target EXIT curves of the amalgamated "MSDSD $-\mathrm{URC}_{s}-\mathrm{IrCC}_{s}$ " decoder of the BS, as shown in Fig. 18. Consequently, near-error-free decoding can be realized by the information exchange between the combined "MSDSD ${ }_{s}-\mathrm{URC}_{s}-\mathrm{IrCC}_{s}$ " and "MSDSD $r-\mathrm{IrCC}_{r}$ " decoders. Note that this cannot be achieved by simply using one of $17 \mathrm{IrCC}_{r}$ subcodes having the same code rate, as observed in Fig. 18, owing to the absence of an open EXIT tunnel.

\section{The Performance of the CRO Near-Capacity DDF-Aided Cooperativey System}

We have now completed the low-complexity near-capacity system design conceived for the single-relay-aided cooperative system. The corresponding Monte-Carlo simulation based decoding trajectory is now plotted in Fig. 18, which reaches the $(1.0,1.0)$ point of the EXIT chart, indicating the achievement of decoding convergence to an infinitesimally low BER at near-capacity SNRs for the IrDD coding scheme proposed

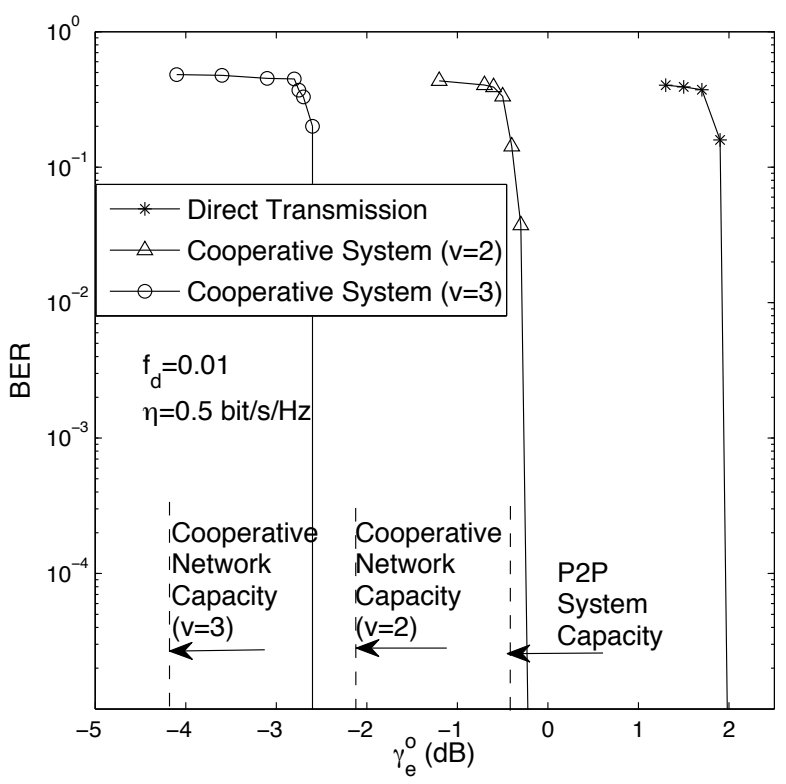

Fig. 19. Achievable BER performance of the near-capacity designed singlerelay-assisted cooperative system.

in Section VI-A2. In Fig. 19, the BER curves of a 'welldesigned' cooperative systems in Scenarios I and II of Table V are portrayed in comparison to that of the conventional nearcapacity point-to-point transmission based system having an identical bandwidth efficiency of $\eta=0.5 \mathrm{bits} / \mathrm{s} / \mathrm{Hz}$. Upon using the near-capacity system design of Section VI-B, the proposed IrDD coding scheme becomes capable of performing within about $1.8 \mathrm{~dB}$ from the corresponding single-relay-aided DDF cooperative system's DCMC capacity in both Scenarios I and II. Observe in Fig. 19 that the single-relay-aided DDF system is capable of outperforming the conventional directtransmission based system by about $2.2 \mathrm{~dB}$ for a given BER target of $10^{-5}$ in Scenario II associated with $v=2$, when both systems are designed to approach their corresponding theoretical maximum transmission rate. By contrast, in Scenario I associated with $v=3$, the single-relay-aided cooperative system becomes capable of even more significantly outperforming the direct-transmission based system, requiring an overall transmit power, which is about $4.6 \mathrm{~dB}$ lower than that needed by the latter to achieve an infinitesimally low BER, while maintaining a bandwidth efficiency of $\eta=0.5 \mathrm{bits} / \mathrm{s} / \mathrm{Hz}$.

\section{Conclusions, Design Guidelines And Future RESEARCH}

\section{A. Summary and Conclusions}

Non-coherent detection aided differential transmission techniques were advocated in this treatise as an appealing, practically implementable candidate for user-cooperation assisted systems. In order to enhance the differentially modulated cooperative system's robustness and flexibility in coping with the hostile time-variant wireless channel, a MCMS joint processing based MSDSD was devised for differentially modulated cooperative systems in Section III, which was also shown in Section VI to be a capacity-achieving SISO detector. The 
crucial role of resource allocation and the need for flexible cooperative protocols was emphasized using different cooperative strategies in Section IV. In the light of the complementary properties of the DAF and DDF schemes, a resource-optimized hybrid cooperative system was proposed to further enhance the achievable performance. Furthermore, due to the half-duplexrelaying-induced multiplexing loss, recent research [30] has revealed that the AF-based cooperative system is typcially inferior to the simple direct-transmission system in terms of the achievable spectral efficiency. Against this background, investigations on the capacity of the DDF-based system were carried out in Section V, which indicated that in typical urban areas the optimized-TRA-based cooperative system is capable of attaining a significantly higher spectral efficiency in the low-SNR range in comparison to its direct-transmission based counterpart. This theoretical result was further evidenced by the pratical design of a CRO near-capacity DDF-aided system in Section VI.

\section{B. Design Guidelines}

- MIMOs circumvent the capacity/power limitation of classic single-antenna-aided systems.

- Regretfully, the MIMO-capacity degrades in the presence of correlated shadow-fading. Hence the single-antennabased mobiles, which are sufficiently far apart may form a VAA to circumvent this limitation with the aid of cooperation, as depicted in Fig. 4.

- Another challenge in the design of MIMOs is their channel estimation, since they require the estimation of $\left(N_{\mathrm{Tx}} \times N_{\mathrm{Rx}}\right)$ links, which is extremely demanding both in terms of its computational requirements as well as in terms of its potentially excessive pilot overhead. This is particularly so for high Doppler frequencies. These two factors may lead to a performance erosion, which may be mitigated with the aid of low-complexity noncoherent detection aided MIMOs dispensing with channel estimation.

- Indeed, coherent-detection aided VAAs would be even more challanging to design than their classic MIMO counterparts relying on co-located elements, since it is somewhat unrealistic to expect the low-complexity, lightweight MSs to estimate each other's channels, let alone the associated data-security aspects of potential eavesdropping... This motivates the design of non-coherent cooperative or VAAs, as advocated in the paper.

- However, the widely recognized impediment of lowcomplexity non-coherent detection is its typical $3 \mathrm{~dB}$ performance loss and the potential BER-floor experienced in case of high Doppler frequencies.

- Although this BER-floor may be eliminated with the aid of ML-MSDD, this 'Doppler-resilient' performance is achieved at a cost, since the ML-detection complexity increases exponentially with the detection-window width $N_{\text {wind }}$.

- Fortunately, this complexity problem may be remedied with the aid of near-ML differential sphere detection, namely the MSDSD devised in Section III.

- The family of cooperation-aided VAAs redefines a number of classic wireless communications problems, such as their resource-allocation for example, which was addressed in Section IV in the context of cooperative relay selection, power-control, AF versus DF relay-mode activation, etc.

- However,cooperation is achieved at the cost of a potential throughput loss owing to the data-exchange required by the cooperative MSs, which are unable to transmit and receive simultaneously.

- The challenges encountered in the DDF-aided system's pratical implementations, such as channel-coding specifically designed for DDF relaying and the avoidance of relaying-induced error propagation were addressed in the context of near-capacity cooperative transceiver architectures. When relying on sophisticated channel-coding and receiver-diversity combiners, the best way to amalgamate the direct link's and the RD link's soft-information is to iteratively exchange extrinsic information between these two links.

\section{Future Research}

Nonetheless, there are numerous open problems associated with the design of the differentially modulated cooperative system, among others the multiuser/multistream interference management seems to be the most critical and challenging problem that has to be solved in order to design other high-efficiency non-orthogonal transmission based cooperative systems, such as the spatial division multiple access (SDMA) based successive relaying scheme of Fig. 1(d). Additionally, scheduling and adaptive rate control is another issue associated with the differentially modulated cooperative systems that has to be studied for the sake of maintaining a high throughput. In the context of differentially modulated cooperative systems relying on non-coherent detection, we may seek solutions dispensing with CSI, while using EXIT-chart-based design techniques [92]. Meanwhile, high-order differentially encoded modulation schemes, such as the star-QAM, and its corresponding advanced non-coherent detection may be also worth investigating for achieving a high-throughput in the context of adaptive modulation aided communications. Finally, the synchronization issues of cooperative systems require substantial attention.

\section{APPENDIX}

Acronyms (See Table VI.)

\section{REFERENCES}

[1] D. Gesbert, M. Shafi, D. shan Shiu, P. J. Smith, and A. Naguib, "From theory to practice: an overview of MIMO space-time coded wireless systems," IEEE J. Sel. Areas Commun., vol. 21, pp. 281-302, Apr. 2003.

[2] J. Mietzner, R. Schober, L. Lampe, W. H. Gerstacker, and P. A. Hoeher, "Multiple-antenna techniques for wireless communications a comprehensive literature survey," IEEE Commun. Surveys Tutorials, vol. 11, pp. 87-105, second quater 2009.

[3] G. J. Foschini, "Layered space-time architecture for wireless communication in fading environments when using multiple antennas," Bell Labs Technical Journal, vol. 2, pp. 41-59, 1996.

[4] G. J. Foschini and M. J. Gans, "On limits of wireless communications in a fading environment when using multiple antennas," Wireless Personal Communications, vol. 6, pp. 311-335, Mar. 1998.

[5] N. Chiurtu, B. Rimoldi, and E. Telatar, "On the capacity of multiantenna Gaussian channels," in Proc. IEEE International Symposium on Information Theory, (Washington, DC), p. 53, June 2001. 
TABLE VI

ACRONYMS

$\begin{array}{lll}\text { AF } & \text { Amplify-and-Forward } & \text { IrDD } \\ \text { AGC } & \text { Automatic Gain Control } & \text { LLR } \\ \text { APA } & \text { Adaptive Power Allocation } & \text { MCMS } \\ \text { APP } & \text { A Posteriori Probability } & \text { MI } \\ \text { BER } & \text { Bit Error Rate } & \text { MIMO } \\ \text { BS } & \text { Base Station } & \text { ML } \\ \text { CDD } & \text { Conventional Differential Detection } & \text { ML-MSDD } \\ \text { CDMA } & \text { Code Division Multiple Access } & \text { MRC } \\ \text { CF } & \text { Compress-and-Forward } & \text { MS } \\ \text { CIR } & \text { Channel Impulse Response } & \text { MSDSD } \\ \text { CRC } & \text { Cyclic Redundancy Check } & \text { P2P } \\ \text { CRO } & \text { Code Rate Optimized } & \text { PDF } \\ \text { CSI } & \text { Channel State Information } & \text { QoE } \\ \text { CUS } & \text { Cooperating User Selection } & \text { RS } \\ \text { DAF } & \text { Differential Amplify-and-Forward } & \text { SD } \\ \text { DDF } & \text { Differential Decode-and-Forward } & \text { SDMA } \\ \text { DF } & \text { Decode-and-Forward } & \text { SISO } \\ \text { DL } & \text { Downlink } & \text { TDMA } \\ \text { DPSK } & \text { Differential Phase-Shift Keying } & \text { TRA } \\ \text { EXIT } & \text { EXtrinsic Information Transfer } & \text { UL } \\ \text { FDMA } & \text { Frequency Division Multiple Access } & \text { URC } \\ \text { IIR } & \text { Infinite Impulse Response } & \text { VAA } \\ \text { IrCC } & \text { Irregular Convolutional Code } & \end{array}$

[6] S. M. Alamouti, "A simple transmit diversity technique for wireless communications," IEEE J. Sel. Areas Commun., vol. 16, pp. 1451-1458, Oct. 1998.

[7] B. Hochwald, T. L. Marzetta, and C. B. Papadias, "A transmitter diversity scheme for wideband CDMA systems based on space-time spreading," IEEE J. Sel. Areas Commun., vol. 19, pp. 48-60, Jan. 2001.

[8] W. F. Su, Z. Safar, and K. J. R. Liu, "Space-time signal design for timecorrelated Rayleigh fading channels," IEEE International Conference on Communications 2003., vol. 5, pp. 3175-3179, May 2003.

[9] W. Su and X. G. Xia, "On space-time block codes from complex orthogonal designs," Wireless Personal Communications, vol. 25, pp. 126, April 2003.

[10] V. Tarokh, H. Jafarkhani, and A. R. Calderbank, "Space-time block codes from orthogonal designs," IEEE Trans. Inf. Theory, vol. 45, pp. 1456-1467, July 1999.

[11] G. D. Golden, C. J. Foschini, R. A. Valenzuela, and P. W. Wolniansky, "Detection algorithm and initial laboratory results using V-BLAST space-time communication architecture," Electronics Letters, vol. 35, pp. 14-16, Jan. 1999.

[12] H. Lee, B. Lee, and I. Lee, "Iterative detection and decoding with an improved v-BLAST for MIMO-OFDM systems," IEEE J. Sel. Areas Commun., vol. 24, pp. 504-513, Mar. 2006.

[13] L. Z. Zheng and D. N. C. Tse, "Diversity and multiplexing: a fundamental tradeoff in multiple-antenna channels," IEEE Trans. Inf. Theory, vol. 49, pp. 1073-1096, May 2003.

[14] R. W. Heath and A. J. Paulraj, "Switching between diversity and multiplexing in MIMO systems," IEEE Trans. Commun., vol. 53, pp. 962 968, June 2005.

[15] T. S. Rappaport, Wireless Communications Principles and Practise. Pearson Education Asia Limited and Publishing House of Electronics Industry, second ed., 2002.

[16] T. Cover and A. E. Gamal, "Capacity theorems for the relay channel," IEEE Trans. Inf. Theory, vol. 25, pp. 572-584, Sept. 1979.

[17] R. Pabst, "Relay-based deployment concepts for wireless and mobile broadband radio," IEEE Commun. Mag., vol. 42, pp. 80-89, Sept. 2004.

[18] D. Soldani and S. Dixit, "Wireless relays for broadband access," IEEE Commun. Mag., vol. 46, pp. 58-66, Mar. 2008.

[19] A. Sendonaris, E. Erkip, and B. Aazhang, "User cooperation diversity. part I: System description," IEEE Trans. Commun., vol. 51, pp. 19271938, Nov. 2003.

[20] A. Sendonaris, E. Erkip, and B. Aazhang, "User cooperation diversity. part II: Implementation aspects and performance analysis," IEEE Trans. Commun., vol. 51, pp. 1939-1948, Nov. 2003.

[21] J. N. Laneman, D. N. C. Tse, and G. W. Wornell, "Cooperative diversity in wireless networks: Efficient protocols and outage behavior," IEEE Trans. Inf. Theory, vol. 50, pp. 3062-3080, Dec. 2004.

[22] K. G. Seddik, A. K. Sadek, W. Su, and K. J. R. Liu, "Outage analysis and optimal power allocation for multinode relay networks," IEEE Signal Process. Lett., vol. 14, pp. 377-380, June 2007.

\author{
Irregular Distributed Differential Coding \\ Log-likelihood Ratio \\ Multiple-Channel Multiple-Symbol \\ Multual Information \\ Multiple-Input Multiple-Output \\ Maximum Likelihood \\ Maximum-Likelihood Multiple-Symbol Differential Detection \\ Maximum Ratio Combining \\ Mobile Station \\ Multiple-Symbol Differential Sphere Detection \\ Point-to-Point \\ Probability Density Function \\ Quality of End-User Experience \\ Relay Station \\ Sphere Detection \\ Spatial Division Multiple Access \\ Soft-Input Soft-Output \\ Time Division Multiple Access \\ Time Resource Allocation \\ Uplink \\ Unity-Rate-Code \\ Virtual Antenna Array
}

[23] G. Farhadi and N. Beaulieu, "Fixed relaying versus selective relaying in multi-hop diversity transmission systems," IEEE Trans. Commun., vol. 58, pp. 956-965, Mar. 2010.

[24] W. W. Peterson and D. T. Brown, "Cyclic codes for error detection," Proc. Institute of Radio Engineers, vol. 49, pp. 228-235, Jan. 1961.

[25] T. E. Hunter and A. Nosratinia, "Diversity through coded cooperation," IEEE Trans. Wireless Commun., vol. 5, pp. 283-289, Feb. 2006.

[26] B. Zhao and M. C. Valenti, "Distributed turbo coded diversity for relay channel," Electronics Letters, vol. 39, pp. 786-787, May 2003.

[27] M. Janani, A. Hedayat, T. E. Hunter, and A. Nosratinia, "Coded cooperation in wireless communications: space-time transmission and iterative decoding," IEEE Trans. Wireless Commun., vol. 52, pp. 362371, Feb. 2004.

[28] G. Kramer, M. Gastpar, and P. Gupta, "Cooperative strategies and capacity theorems for relay networks," IEEE Trans. Wireless Commun., vol. 51, pp. 3037-3063, Sept. 2005.

[29] S. Simoens, O. Muoz-Medina, J. Vidal, and A. D. Coso, "Compress-andforward cooperative mimo relaying with full channel state information," IEEE Trans. Wireless Commun., vol. 58, pp. 781-791, Feb. 2010.

[30] S. S. Ikki, M. Uysal, and M. H. Ahmed, "Performance analysis of incremental-best-relay amplify-and-forward technique," IEEE Global Telecommunications Conference 2009 (GLOBECOM'09), Nov. 2009.

[31] Y. Wu, P. A. Chou, and S.-Y. Kung, "Information exchange in wireless networks with network coding and physical-layer broadcast," 39th Conference on Information Science and Systems, Mar. 2005.

[32] P. Larsson, N. Johansson, and K.-E. Sunell, "Coded bi-directional relaying," IEEE 63rd Vehicular Technology Conference, pp. 851-855, May 2006.

[33] P. Popovski and H. Yomo, "The anti-packets can increase the achievable throughput of a wireless multi-hop network," IEEE International Conference on Communications, vol. 9, pp. 3885-3890, 2006.

[34] H. J. Yang, K. Lee, and J. Chun, "Zero-forcing based two-phase relaying," IEEE International Conference on Communications, pp. 5224$5228,2007$.

[35] J. Zhao, M. Kuhn, A. Wittneben, and G. Bauch, "Self-interference aided channel estimation in two-way relaying systems," IEEE Global Telecommunications Conference, pp. 3659-3664, 2008.

[36] Y. Fan, C. Wang, J. Thompson, and H. V. Poor, "Recovering multiplexing loss through successive relaying using repetition coding," IEEE Trans. Wireless Commun., vol. 6, pp. 4484-4493, Dec. 2007.

[37] B. Hassibi and B. M. Hochwald, "High-rate codes that are linear in space and time," IEEE Trans. Inf. Theory, vol. 48, pp. 1804-1824, July 2002.

[38] J. N. Laneman and G. W. Wornell, "Distributed space-time coded protocols for exploiting cooperative diversity in wireless networks," IEEE Trans. Inf. Theory, vol. 49, pp. 2415-2425, Oct. 2003.

[39] R. U. Nabar, H. Bolcskei, and F. W. Kneubuhler, "Fading relay channles: performance limits and space-time signal design," IEEE J. Sel. Areas Commun., vol. 22, pp. 1099-1109, Aug. 2004. 
[40] S. Yiu, R. Schober, and L. Lampe, "Distributed space-time block coding," IEEE Trans. Commun., vol. 54, pp. 1195-1206, 2006.

[41] B. Sirkeci-Mergen and A. Scaglione, "Randomized space-time coding for distributed cooperative communication," IEEE Trans. Signal Process., vol. 55, pp. 5003-5017, 2007.

[42] Q. Huang, M. Ghogho, J. Wei, and P. Ciblat, "Practical timing and frequency synchronization for ofdm-based cooperative systems," IEEE Trans. Wireless Commun., vol. 58, pp. 3706-3716, July 2010.

[43] A. S. Ibrahim and K. J. R. Liu, "Mitigating channel estimation error with timing synchronization tradeoff in cooperative communications," IEEE Trans. Signal Process., vol. 58, pp. 337-348, Jan. 2010.

[44] Y. Wu and M. Patzold, "Performance analysis of cooperative communication systems with imperfect channel estimation," IEEE International Conference on Communications (ICC'09), June 2009.

[45] S. Han, S. Ahn, E. Oh, and D. Hong, "Effect of channel-estimation error on ber performance in cooperative transmission," IEEE Trans. Veh. Technol., vol. 58, pp. 2083-2088, May 2009.

[46] W. Chen, L. Dai, K. B. Letaief, and Z. Cao, "A unified cross-layer framework for resource allocation in cooperative networks," IEEE Trans. Wireless Commun., vol. 7, pp. 3000-3012, Aug. 2008.

[47] W. Shim, Y. Han, and S. Kim, "Fairness-aware resource allocation in a cooperative ofdma uplink system," IEEE Trans. Wireless Commun., vol. 59, pp. 932-939, Feb. 2010.

[48] T. Himsoon, W. P. Siriwongpairat, W. Su, and K. J. R. Liu, "Differential modulations for multinode cooperative communications," IEEE Trans. Signal Process., vol. 56, pp. 2941-2956, July 2008.

[49] T. Himsoon, W. P. Siriwongpairat, W. Su, and K. J. R. Liu, "Differential modulation with threshold-based decision combining for cooperative communications," IEEE Trans. Signal Process., vol. 55, pp. 3905-3923, July 2007.

[50] W. Su, F. Chen, D. A. Pados, and J. D. Matyjas, "The outage probability and optimum power assignment for differential amplify-and-forward relaying," IEEE International Conference on Communications, pp. 1-5, May 2010.

[51] Q. Zhao and H. Li, "Performance of differential modulation with wireless relays in Rayleigh fading channels," IEEE Commun. Lett. vol. 9, pp. 343-345, Apr. 2005.

[52] Q. Zhao and H. Li, "Differential modulation for cooperative wireless systems," IEEE Trans. Signal Process., vol. 55, pp. 2273-2283, May 2007.

[53] T. Cui, F. Gao, and C. Tellambura, "Differential modulation for two-way wireless communications: a perspective of differential network coding at the physical layer," IEEE Trans. Commun., vol. 57, pp. 2977-2987, Oct. 2009

[54] L. Hanzo, Y. Akhtman, L. Wang, and M. Jiang, MIMO-OFDM for LTE, WIFI and WIMAX: Coherent versus Non-Coherent and Cooperative Turbo-Transceivers. John Wiley and IEEE Press, 2010.

[55] J. G. Proakis, Digital Communications. 4th edition, New York, NY: Mc-Graw-Hill, 2000.

[56] G. Wang, Y. Zhang, and M. Amin, "Differential distributed space-time modulation for cooperative networks," IEEE Trans. Wireless Commun., vol. 5, pp. 3097-3108, Nov. 2006.

[57] Y. Jing and H. Jafarkhani, "Distributed differential space-time coding for wireless relay networks," IEEE Trans. Commun., vol. 56, pp. 1092 1100 , July 2008.

[58] F. Oggier and E. Lequeu, "Differential distributed cayley space-time codes," IEEE Trans. Wireless Commun., vol. 8, pp. 3808-3814, July 2009.

[59] S. Yiu, R. Schober, and L. Lampe, "Differential distributed spacetime block coding," IEEE Pacific Rim Conference on Communications, Computers and Signal Processing, pp. 53-56, 2005.

[60] Y. Liang and V. V. Veeravalli, "Capacity of noncoherent time-selective rayleigh-fading channels," IEEE Trans. Inf. Theory, vol. 50, pp. 30953110, Dec. 2004.

[61] T. Himsoon, W. Su, and K. J. R. Liu, "Differential transmission for amplify-and-forward cooperative communications," IEEE Signal Process. Lett., vol. 12, pp. 597-600, Sept. 2005.

[62] D. G. Brennan, "Linear diversity combining techniques," Proc. IEEE, vol. 91, pp. 331-356, Feb. 2003.

[63] M. K. Simon and M. S. Alouini, "A unified approach to the probability of error for noncoherent and differentially coherent modulations over generalized fading channels," IEEE Trans. Commun., vol. 46, pp. 16251638, Dec. 1998

[64] L. Wang and L. Hanzo, "The resource-optimized differentially modulated hybrid $\mathrm{AF} / \mathrm{DF}$ cooperative cellular uplink using multiple-symbol differential sphere detection," IEEE Signal Process. Lett., vol. 16, pp. 965-968, Nov. 2009.
[65] H. Ochiai, P. Mitran, and V. Tarokh, "Design and analysis of collaborative diversity protocols for wireless sensor networks," in IEEE 60th Vehicular Technology Conference, vol. 7, no. 4, pp. 4645-4649, 2004.

[66] L. Wang and L. Hanzo, "The amplify-and-forward cooperative uplink using multiple-symbol differential sphere-detection," IEEE Signal Process. Lett., vol. 16, pp. 913-916, Oct. 2009.

[67] U. Fincke and M. Pohst, "Improved methods for calculating vectors of short length in a lattice, including a complexity analysis," Mathematics of Computation, vol. 44, pp. 463-471, April 1985.

[68] E. Viterbo and J. Boutros, "A universal lattice code decoder for fading channels," IEEE Trans. Inf. Theory, vol. 45, pp. 1639-1642, July 1999.

[69] L. Lampe, R. Schober, V. Pauli, and C. Windpassinger, "Multiplesymbol differential sphere decoding," IEEE Trans. Commun., vol. 12, pp. 1981-1985, Dec. 2005.

[70] D. Divsalar and M. K. Simon, "Multiple-symbol differential detection of MPSK," IEEE Trans. Commun., vol. 38, pp. 300-308, Mar. 1990.

[71] D. Divsalar and M. K. Simon, "Maximum-likelihood differential detection of uncoded and trellis-coded amplitude phase modulation over awgn and fading channels-metrics and performance," IEEE Trans. Commun., vol. 42, pp. 76-89, Jan. 1994.

[72] R. A. Horn and C. R. Johnson, Matrix Analysis. Cambridge University Press, 1985.

[73] M. O. Damen, K. Abed-Meraim, and J. C. Belfiore, "Generalised sphere decoder for asymmetrical space-time communication architecture," Electronics Letters, vol. 36, pp. 166-167, Jan. 2000.

[74] T. Cui and C. Tellambura, "An efficient generalized sphere decoder for rank-deficient MIMO systems," IEEE Commun. Lett., vol. 9, pp. 423425, May 2005.

[75] J. Jalden and B. Ottersten, "On the complexity of sphere decoding in digital communications," IEEE Trans. Signal Processing, vol. 53, pp. 1474-1484, 2005 .

[76] J. Akhtman and L. Hanzo, "An optimized-hierarchy-aided maximum likelihood detector for MIMO-OFDM," IEEE 63rd Vehicular Technology Conference, VTC 2006-Spring, vol. 3, pp. 1526-1530, 2006.

[77] I. Motedayen-Aval, A. Krishnamoorthy, and A. Anastasopoulos, "Optimal joint detection/estimation in fading channels with polynomial complexity," IEEE Trans. Inf. Theory, vol. 53, pp. 209-223, 2007.

[78] V. Pauli, L. Lampe, R. Schober, and K. Fukuda, "Multiple-symbol differential detection based on combinatorial geometry," IEEE Trans. Commun., vol. 56, pp. 1596-1600, 2008.

[79] V. Pauli and L. Lampe, "Multiple-symbol differential sphere decoding for unitary space-time modulation," IEEE Global Telecommunications Conference, vol. 3, p. 6, Nov. 2005.

[80] V. Pauli, L. Lampe, and R. Schober, “"Turbo DPSK” using soft multiplesymbol differential sphere decoding," IEEE Trans. Inf. Theory, vol. 52, no. 4, pp. 1385-1398, 2006.

[81] V. Pauli and L. Lampe, "On the complexity of sphere decoding for differential detection," IEEE Trans. Inf. Theory, vol. 53, pp. 1595-1603, Apr. 2007.

[82] V. Pauli, L. Lampe, and J. Huber, "Differential space-frequency modulation and fast 2-d multiple-symbol differential detection for MIMOOFDM," IEEE Trans. Veh. Technol., vol. 57, pp. 297-310, Jan. 2008.

[83] T. M. Cover and J. A. Thomas, Elements of Information Theory. Second Edition, John Wiley \& Sons, Inc., 2006.

[84] R. R. Chen, R. Koetter, U. Madhow, and D. Agrawal, "Joint noncoherent demodulation and decoding for the block fading channel: a practical framework for approaching shannon capacity," IEEE Trans. Commun., vol. 51, pp. 1676-1689, Oct. 2003.

[85] A. Host-Madsen and J. Zhang, "Capacity bounds and power allocation for wireless relay channel," IEEE Trans. Inf. Theory, vol. 51, pp. 20202040, June 2005.

[86] M. Tüchler and J. Hagenauer, "Exit charts of irregular codes," in Proceedings of Conference on the Information Science and Systems [CDROM], Princeton University, pp. 20-22, 2002.

[87] L. Hanzo, O. Alamri, M. El-Hajjar, and N. Wu, Near-Capacity MultiFunctional MIMO Systems: sphere-packing, iterative detection, and cooperation. First Edition, John Wiley \& Sons Ltd and IEEE Press, 2009.

[88] M. Tüchler, "Design of serially concatenated systems depending on the block length," IEEE Trans. Commun., vol. 52, pp. 209-218, Feb. 2004.

[89] A. Ashikhmin, G. Kramer, and S. ten Brink, "Extrinsic information transfer functions: model and erasure channel properties," IEEE Trans. Inf. Theory, vol. 50, pp. 2657-2673, Nov. 2004.

[90] S. ten Brink, "Convergence behavior of iteratively decoded parallel concatenated codes," IEEE Trans. Commun., vol. 49, pp. 1727-1737, Oct. 2001 . 
[91] J. Kliewer, S. X. Ng, and L. Hanzo, "Efficient computation of EXIT functions for non-binary iterative decoding," IEEE Trans. Communications, vol. 54, pp. 2133-2136, Dec. 2006.

[92] S. Ibi, T. Matsumoto, R. Thoma, S. Sampei, and N. Morinaga, "Exit chart-aided adaptive coding for multilevel bicm with turbo equalization in frequency-selective mimo channels," IEEE Trans. Vehicular Technology, vol. 56, pp. 3757-3769, Nov. 2007.

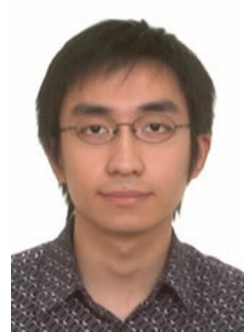

Li Wang (S'09-M'10) was born in Chengdu, China, in 1982. He received his BEng degree in information engineering from Chengdu University of Technology (CDUT), Chengdu, China, in 2005 and his MSc degree with distinction in radio frequency communication systems from the University of Southampton, UK, in 2006. Between October 2006 and January 2010 he was a PhD student in the Communications Group, School of Electronics and Computer Science, University of Southampton, and participated in the Delivery Efficiency Core Research Programme of the Virtual Centre of Excellence in Mobile and Personal Communications (Mobile VCE). He was awarded a joint EPSRC and Mobile VCE full scholarship. Upon the completion of his PhD in January 2010 he conducted research as a senior research fellow in the School of Electronics and Computer Science at the University of Southampton. Currently, he is involved in the Project \#7 of the Indian-UK Advanced Technology Centre (IU-ATC): advanced air interface technique for MIMO-OFDM and cooperative communications. His research interests include space-time processing, channel coding, multi-user detection, non-coherent transmission techniques, iterative detection, EXIT-chart-aided system design, MIMO-OFDM and cooperative systems.

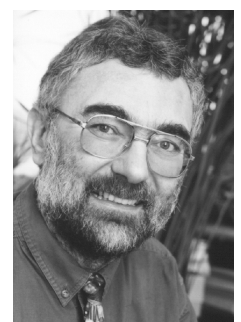

Lajos Hanzo (http://www-mobile.ecs.soton.ac.uk) FREng, FIEEE, FIET, DSc received his degree in electronics in 1976 and his doctorate in 1983. In 2009 he was awarded the honorary doctorate "Doctor Honaris Causa" by the Technical University of Budapest. During his 35-year career in telecommunications he has held various research and academic posts in Hungary, Germany and the UK. Since 1986 he has been with the School of Electronics and Computer Science, University of Southampton, UK, where he holds the chair in telecommunications. He has co-authored 20 John Wiley/IEEE Press books on mobile radio communications totalling in excess of 10000 pages, published 1200+ research entries at IEEE Xplore, acted as TPC Chair of IEEE conferences, presented keynote lectures and been awarded a number of distinctions. Currently he is directing an academic research team, working on a range of research projects in the field of wireless multimedia communications sponsored by industry, the Engineering and Physical Sciences Research Council (EPSRC) UK, the European IST Programme and the Mobile Virtual Centre of Excellence (VCE), UK. He is an enthusiastic supporter of industrial and academic liaison and he offers a range of industrial courses. He is also a Governor of the IEEE VTS Since 2008 he has been the Editor-in-Chief of the IEEE Press and since 2009 a Chaired Professor also at Tsinghua University, Beijing. For further information on research in progress and associated publications please refer to http://www-mobile.ecs.soton.ac.uk 